ISSN: 0213-2060

DOI: https://doi.org/10.14201/shhme2020381195220

\title{
IL PORTO E LA STRADA. SPUNTI DI RIFLESSIONE SULLA TRADIZIONE CONCERNENTE LA FONDAZIONE DI MANFREDONIA
}

\author{
The Harbor and the Street. Insights on the Tradition Concerning the Manfredonia's \\ Foundation
}

\begin{abstract}
Antonio Pio DI COSMO
Dipartimento di Storia. Facoltà di Scienze Ecclesiastiche Orientali, Pontificio Istituto Orientale. Piazza di S. Maria Maggiore, 7.00185 ROMA (Italia). C. e.: apiocosmo@outlook.it. ORCID: https://orcid.org/0195-2202$2314-4520$
\end{abstract}

Recibido: 2019-08-24

Revisado: 2020-02-05

Aceptado: 2020-05-04

RIASSUNTO: La presente ricerca analizza la tradizione concernente la fondazione di Manfredonia e sottopone ad una serrata critica il suo contenuto. Vengono poi approfondite le ragioni che portano all'auge Manfredonia. La città sembra così costruirsi attorno ai nuclei del porto e della strata magna. Si tentano poi di interpretare le diverse tradizioni circolanti sulla nascita di Manfredonia.

Parole chiave: Manfredonia; Manfredi; Fondazione; Porto; Strata magna.

RESUMEN: El presente estudio analiza la tradición relativa a la fundación de Manfredonia y somete su contenido a una profunda crítica. Además, se analizan las razones que conducen al auge de Manfredonia. La ciudad parece, así, construirse en torno a los núcleos del puerto y de la strata magna. Por último, se intenta interpretar las distintas tradiciones que circulan acerca del nacimiento de Manfredonia.

Palabras clave: Manfredonia; Manfredi; Fundación; Puerto; Strata magna.

ABSTRACT: This research analyzes the tradition, which concerning the foundation of Manfredonia and criticizes its content. The research also deepens the reasons, that caused the rise of Manfredonia, which was built around the harbor and the strata magna. Finally, it interpreted the different traditions circulating about the birth of Manfredonia.

Keywords: Manfredonia; Manfred; Foundation; Harbor; Strata magna. 
SOMMARIO: 0 Introduzione. 1 Siponto ed il Casale Syponti: problematiche di continuità abitativa. 2 Il porto come catalizzatore demico: un fattore di antropizzazione. 3 Il sito di fondazione ed i problemi concernenti la costruzione dell'identità cittadina. 4 L'intitolazione a Manfredi e la rielaborazione della memoria. 5 Conclusioni. 6 Riferimenti bibliografici.

\section{INTRODUZIONE}

La presente ricerca procede dalle problematiche poste dalla tradizione concernente la fondazione di Manfredonia, che non sembra essersi verificata come un atto "ufficiale», dipendente dalla volontà regia o comunque signorile. Tale incertezza ha permesso ad alcuni studiosi locali di «azzerarlo», almeno come evento unico e solenne. L'investigazione è perciò volta a migliorare la comprensione del «carattere» di tale atto, se mai avvenuto, in uno con le finalità politiche e logistiche che presuppone ${ }^{1}$.

I Diurnali di Spinelli offrono una serie di informazioni molto particolareggiate: Manfredonia viene così denominata da Manfredi (1232-1266 d.C.), re e fondatore, il quale è presente alla posa della prima pietra il 23 di aprile del 1256 e si è pure preoccupato di delineare personalmente la pianta cittadina ${ }^{2}$.

Eppure, queste informazioni non sembrano convincere. È noto che i Diurnali di Spinelli costituiscono un testo problematico e controverso ${ }^{3}$. Nonostante ciò, le informazioni riguardanti le origini di Manfredonia sembrano convincere alcuni eruditi dei secc. XVII-XVIII come Pompeo Sarnelli ${ }^{4}$ ed Ughelli ${ }^{5}$. Nondimeno persuadono in tempi più recenti sia Giunta ${ }^{6}$, sia Serricchio. Quest'ultimo argomenta lungamente tale dato: in quell'anno Manfredi è in zona per praticare la caccia nel Bosco di Incoronata. Ritiene

1 Palumbo, Pier Fausto. «La fondazione di Manfredonia». Archivio Storico Pugliese, 1953, vol. IV, p. 371

Spinelli, Matteo. Diurnali, Vigo G. (ed.). Napoli: G. Dura, 1872, pp. 30-33; 40.

Alcuni studiosi credono che il testo costituisca un falso databile al sec. xvi. Altrimenti, se lo si considera autentico, non è possibile sottovalutare la semplicistica azione di quei copisti del xvI sec. che, nel tentativo di ordinare gli annotamenti spinelliani, lo hanno adulterato con rilevanti errori cronologici. Sulla relativa questione cfr. Zazzaretta, Alessandro. «Sui Diurnali di Matteo Spinelli. Premessa per un riesame della questione spinelliana». Archivio Storico Pugliese, 1970, vol. XXIII, pp. 199-214; De Troia, Giuseppe. "Gli annotamenti di Matteo Spinelli da Giovinazzo: frammenti di cronaca del XIII secolo, osservazioni critiche». La Capitanata, 2001, vol. XXVIII, pp. 167-205; Palmieri, Stefano. «Bartolommeo Capasso e l'edizione delle fonti storiche napoletane». Napoli Nobilissima, 2001, vol. III, pp. 147-162; CARLuccI, Rosanna. «I Diurnali di Matteo da Giovinazzo: una Cronaca del XIII secolo?». Studi Bitontini, 2003, vol. LXXV, pp. 41-58; STIPo, Pasquale. I "Notamenti" di Messer Matteo Spinelli da Giovenazzo, tra vero e falso, frammenti di cronaca del $13^{\circ}$ secolo (I Diurnali). Bisceglie: Eurografica, 2013.

$4 \quad$ Sarnelli, Pompeo. Cronologia De’Vescovi et Arcivescovi Sipontini. Manfredonia: Stamperia Arcivescovile, 1680 .

5 Ughelli, Ferdinando. Italia Sacra. Venezia: Forni, 1721, pp. 809-810.

6 Giunta, Francesco. «Manfredi e Manfredonia». In Giunta, Francesco (ed.). La coesistenza nel Medioevo. Bari: Dedalo, 1968, p. 12; Serricchio, Cristanziano. "Manfredi e la fondazione di Manfredonia». Archivio Storico Pugliese, 1972, vol. XXV, p. 486. 
poi verosimile il riferimento a Marino Capece, quale Commessionario pe mare e pe terra ${ }^{7}$, poiché è certo che dal 1263 Manfredi nomina lo zio materno, il conte Manfredi Maletta (1232-1310 d.C.) e Gran Camerlengo del Regno, responsabile dei lavori concernenti la fortificazione del nucleo demico. Anche De Troia è persuaso dalla plausibilità della data ${ }^{8}$ e, persino, Filangieri di Candida dà per buono quel racconto, senza preoccuparsi troppo dei problemi filologici del testo, lasciando però un qualche margine al 1259, quale probabile data di posa della prima pietra ${ }^{9}$.

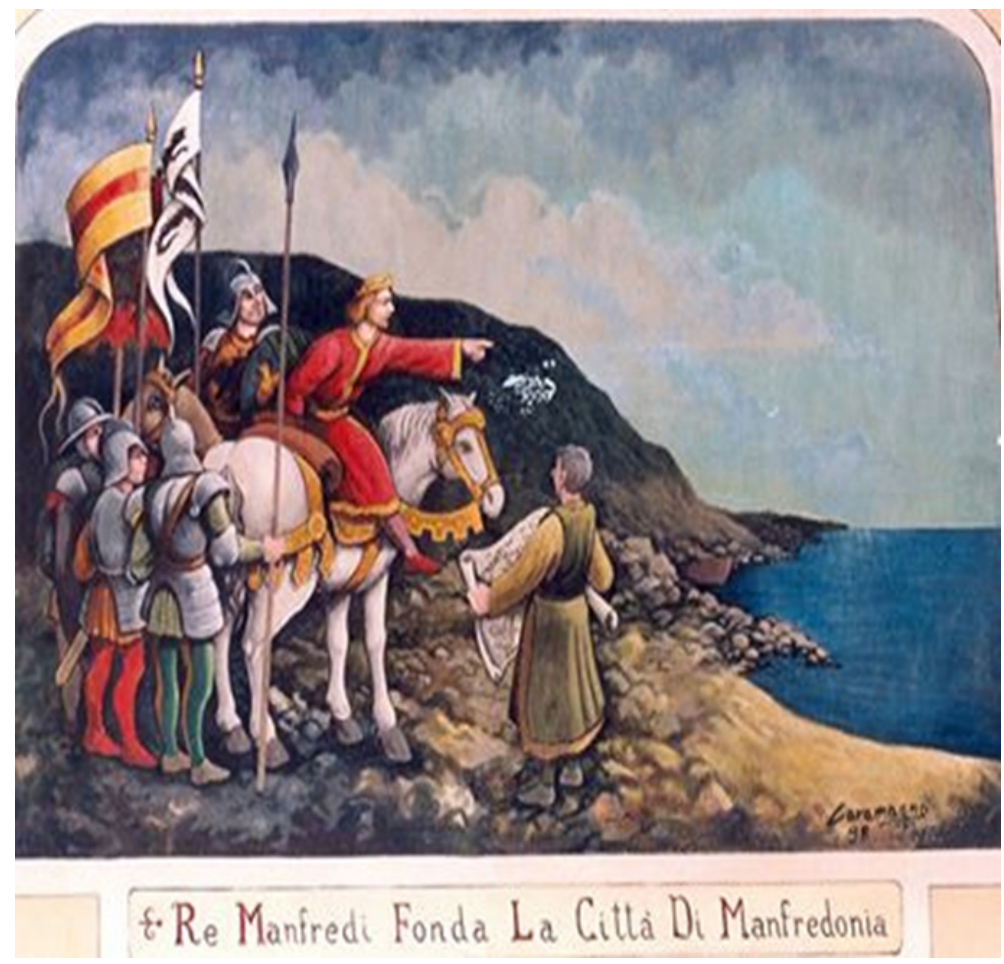

Figura 1. Manfredi indica il sito di Manfredonia, Caramagno, pittura a secco, 2010, Manfredonia.

7 Giungono differenti lezioni circa il nome di tale commissario. Il codice Gesualdini lo definisce Marino Capece, il napoletano invece Marco Capece, il codice Guelferbitano altresì lo addita come Mario Cape$c e$. Tale discordanza secondo De Troia è dovuta al fatto che il suo nome nel manoscritto originario è riportato con la sigla Mãro. Cfr. De Troia, «Gli annotamenti», pp. 186-187.

8 De Troia, Giuseppe. Dalla distruzione di Siponto alla fortificazione di Manfredonia. Fasano: Schena, 1985, pp. 60-61, 69-70; De TroiA, «Gli annotamenti», pp. 167-205.

9 Filangieri Di Candida, Angerio. «Le fondazioni di città nello sviluppo degli insediamenti del Mezzogiorno». Working Paper, 2007, vol. XII, fasc. 1, pp. 23-24. 
Deve poi considerarsi il 7 novembre 1263. Una data che emerge dal cosiddetto Datum Orte. Il documento emesso dalla cancelleria regia fornisce un dato cronologico certo $^{10}$, che però non sembra indicare la fondazione della città, ma piuttosto un momento in cui la cittadina è in stato di avanzata costruzione.

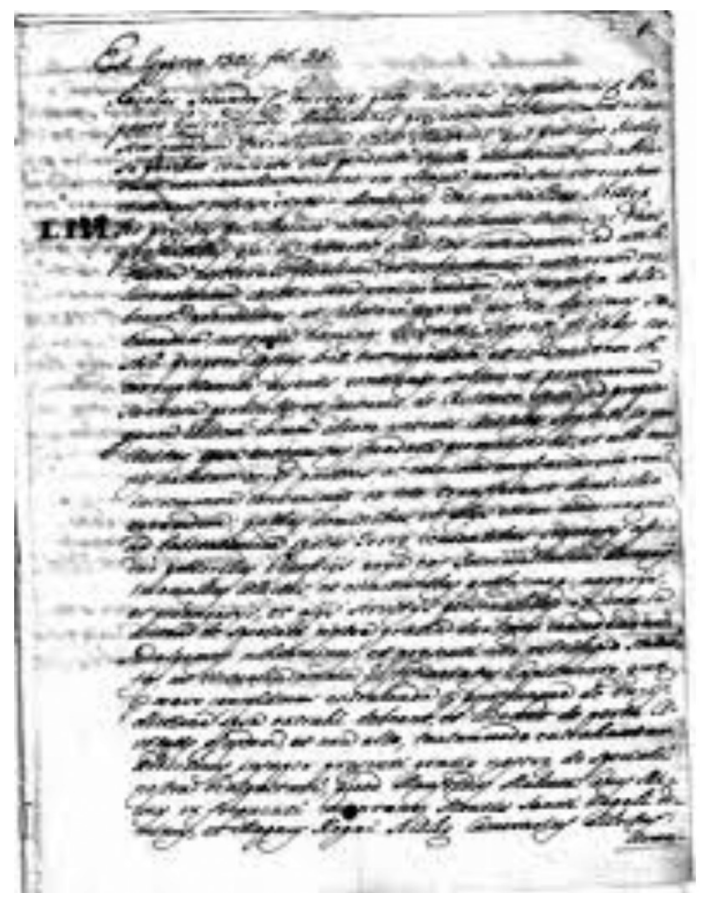

Figura 2. Datum Orte del 1263, inserito in un documento della cancelleria angioina del 1300, il cui testo è ricopiato a metà del xviII secolo. Archivio Storico del Comune di Manfredonia (immagine da Violante, «Da Siponto a Manfredonia», p. 24).

Il 1263 può solo segnare una fase di discontinuità nell'occupazione di Siponto, che è ancora viva e vitale, mentre i suoi abitanti vengono stimolati con incentivi fiscali a prendere dimora nel nuovo centro. L'interessamento regio prova che gli occupanti devono essere un numero talmente consistente da richiedere un provvedimento ad hoc.

Un altro studioso locale, Ognissanti, focalizza la propria attenzione sulla concessione di benefici fiscali attestata dal documento ed offre una potente suggestione: le esenzioni stimolano la nascita e la strutturazione di una classe mercantile locale ${ }^{11}$.

10 Violante, Francesco. "Da Siponto a Manfredonia: note sulla "fondazione"». In Licinio, Raffaele (ed.). Storia di Manfredonia. Il Medioevo. Bari: Edipuglia, 2008, pp. 9-11.

11 Ognissanti, Pasquale. «Il porto di Siponto e di Manfredonia». La Capitanata, 1984-1985, vol. XLII, pp. 9-51; Ognissanti, Pasquale. «La storiografia sipontina». La Capitanata, 1987, vol. XXVI, pp. 1-36. 
Le recenti ricerche archeologiche guidate da Caterina Laganara hanno permesso di comprendere alcuni aspetti della dinamica vitale della Siponto medievale. I rinvenimenti monetali in situ suggeriscono quale termine post quem per la frequentazione un periodo che intercorre fra il Regno di Corrado I, per quel che riguarda le evidenze numismatiche rivenute negli Edifici IX e XII, ed il governo di Manfredi, come testimoniato dai ritrovamenti monetali presso gli Edifici X, XIII e XIV. Le più rilevanti informazioni provengono tuttavia dalle escavazioni degli Edifici X e XIV e dai cospicui gruzzoli di monete ivi scoperti: ben 32 monete nell'edificio X, mentre il XIV ne restituisce solo $20^{12}$. I conii di epoca sveva dell'Edificio X compongono un tesoretto rinvenuto entro uno strato di obliterazione connotato da residui carboniosi, a testimoniare un incendio che ha interessato l'edificio forse sotto Manfredi, in ragione della preponderante presenza di emissioni riconducibili a questo sovrano o, probabilmente, negli anni immediatamente seguenti. Gli esemplari numismatici svevi recuperati nell'Edificio XIV invece sono coniati quasi tutti sotto Corrado II. Si può immaginare che quel gruzzolo venga costituito con poco margine di anticipo rispetto a quello dell'Edificio X. Tali rinvenimenti forniscono un potente indicatore, utile a dimostrare che l'abbandono della città non si è realizzato in tempi stretti. L'occupazione del sito si riduce progressivamente ed entro una cronologia che allo stato attuale degli studi non si riesce a definire con certezza. E se per l'Edificio $\mathrm{X}$ è un evento traumatico come l'incendio, che ha obliterato il gruzzolo di monete, a segnare il momento dell'abbandono, non troppo chiare appaiono le ragioni che spingono gli abitanti dell'Edificio XIV ad allontanarsi senza portare con sé le monete o, almeno, a non tornare per recuperarle.

Stupisce l'assenza di ogni menzione della fondazione di Manfredonia negli storici coevi del Sud Italia come Matteo Paris e Nicolò di Jamsilla. Non è possibile verificare l'effettività di un atto di fondazione unico ed ufficiale, forse perché non è stato percepito dai coevi come «nuova» fondazione. Ragionevolmente questo è connesso, almeno in epoca precoce, a Siponto ed è sentito come sua appendice ${ }^{13}$. Tale ipotesi, tuttavia, appare un po' forzata, poiché i succitati storici sono solitamente molto attenti alle vicende della vita del Regno siciliano. Perciò, non si comprende come possano ignorare un cambiamento degno di rilievo nell'assetto demico della costa levantina. Tale assenza può spiegarsi diversamente: Manfredi si limita ad una mera operazione di sistemazione urbanistica. Segue un più incisivo progetto di fortificazione del nucleo demico già esistente e forse spontaneo presso il nuovo porto, atto a sostituire quello sipontino inutilizzabile ed oggetto di progressivo insabbiamento. Una volta declassato quell'atto «solenne», si può intendere il motivo dell'assenza di ogni ulteriore riferimento alla fondazione nei coevi. Non deve

12 Sarcinelli, Giuseppe. «Moneta reale, moneta di conto: tra fonti materiali e fonti scritte». In Laganara, Caterina (ed.). L'abitare: spazi domestici e quadri di vita. Dalla ricerca archeologica nella Siponto medievale. Foggia: Grenzi Editore, 2012, pp. 121-136, in particolare p. 123; SARCinelli, Giuseppe. «Le monete». In Laganara, Caterina (ed.). Siponto. Archeologia di una città abbandonata nel Medioevo. Foggia: Grenzi Editore, 2011, pp. 197-206; Sarcinelli, Giuseppe. Prima e dopo Manfredi. Monete tra Siponto e Manfredonia nella collezione civica. Foggia: Edizioni del Rosone, 2010, pp. 20-21; LAGANARA, Caterina. «In civitate sipontina... lo spazio urbano e le dinamiche insediative». In Laganara, Siponto. Archeologia di una città, pp. 47-53.

13 Serricchio, «Manfredi», p. 492. 
destare molto clamore un intervento regio volto a regolarizzare l'azione spontanea di soggetti che si trasferiscono presso quel nuovo porto, sistematizzando l'occupazione del suolo entro lo schema delle cosiddette terrae novae.

\section{Siponto ed il CASALE SyPONTI: PRoblematiche di Continuità ABitativa}

Le fonti offrono un'ulteriore suggestione e suggeriscono un presunto sdoppiamento di Siponto, che contrappone il sito della cattedrale all'esistenza di un ulteriore nucleo abitativo di incerta ubicazione, denominato Casale Syponti ${ }^{14}$. Siamo di fronte all'esistenza di un aggregato demico da cui sviluppa Manfredonia? O si attesta una fase di decadenza del tessuto urbano di Siponto? Le informazioni pervenuteci non permettono di intendere quale sia la relazione tra il casale e la «vecchia» Siponto.

Sappiamo da un documento del 1154 che la vita di Siponto procede in modo normale. Un successivo atto, il n. 39 del Regesto di San Leonardo, datato al luglio 1155 attesta il deterioramento della trama urbana, tanto da definirla: civitas diruta $^{15}$. Fonte che Camobreco posticipa al 1156. La significativa alterazione può essere forse ricondotta ad un diverso fenomeno. Martin ha suggerito che questo mutamento in peius è forse dovuto allo spostamento degli abitanti per scelte di carattere produttivo, come la ripresa da parte di un certo numero di loro dell'allevamento transumante ${ }^{16}$. È stato pure postulato che tale fenomeno possa essere conseguenza di un evento sismico, ma il fatto che la cattedrale sia funzionale, permette di escluderlo del tutto. Ciò suggerisce una causa diversa, come la guerra fra re Guglielmo I ed il basileus Manuele I Comneno, che appoggia Roberto conte di Loretello e i baroni ribelli.

Un successivo documento, il n. 41 del Regesto di San Leonardo, datato all'ottobre del 1156 attesta che Siponto perdura in rovina ${ }^{17}$. Il testo viene rogato con l'intestazione che segue il sistema di datazione bizantino, con menzione del nome dell'imperatore Manuele Comneno. Ciò rileva l'esistenza di «sacche» filobizantine, nonostante i pesanti interventi militari dei normanni sul territorio nella guerra contro i romano orientali. Un'informazione che ha suggestionato De Troia ${ }^{18} \mathrm{e}$ lo ha indotto a retrodatare l'atto all'ottobre 1155.

La retrodatazione, se dimostrata corretta, unita alla posticipazione del documento n. 39 del citato Regesto, lascia pensare che almeno dall'ottobre del 1155 Siponto è in rovina. Forse perché oggetto di una spedizione punitiva ad opera di Asclettino, che

14 Martin, Jean Marie. Les actes de l'abbaye de Cava concernant le Gargano. Bari: Società di Storia Patria per la Puglia, 1994, pp. 138-139.

15 Regesto di San Leonardo di Siponto. Camobreco, Fortunato (ed.). Regesta Chartarum Italiae 10. Roma: Istituto Storico Italiano, 1913, doc. n. 39, 1155, pp. 24-25; vedi anche doc. n. 36, pp. 22-23; cfr. Martin, Jean Marie. «La città di Siponto nei secoli XI-XIII». In Houben, Hans (ed.). San Leonardo di Siponto. Cella monastica, canonica, "domus Theutonicorum". Atti del Convegno internazionale, Manfredonia, 18-19 marzo 2005. Galatina: Congedo Editore, 2006, pp. 30-31.

${ }_{16}$ Martin, Jean Marie. La Pouille du VI au XII siècle. Roma: École Française de Rome, 1993, pp. 84-85, nota 94; pp. 107-108.

17 Regesto di San Leonardo di Siponto, doc. n. 41, 1155, pp. 27-28.

18 De Troia, Dalla distruzione, pp. 60-61; 69-70. 
sappiamo essere nel maggio 1155 impegnato nell'assedio di Benevento. Questi poco dopo si dirige verso nord, dove il 30 maggio da alle fiamme Tricolena presso Ferentino. Facendo salva la datazione riportata da tali atti e reputando attendibile quanto attestato dal documento n. 39 del Regesto, appare ragionevole sostenere una precoce distruzione di Siponto, che può realizzarsi dal giugno al luglio 1155. Datazione che sembra essere confermata dal silenzio di Giovanni Cinnamo (1145?-1190? d.C.), il quale si preoccupa di raccontare gli eventi bellici solo dal cuore dall'estate del $1155^{19}$. Un'omissione forse dovuta al fatto che l'intervento militare si realizza nelle prime fasi della guerra ed a seguito di una troppo precoce «scaramuccia», che suscita l'ira della monarchia normanna, ma non suggestiona lo storico bizantino.

Un altro documento del 1175 riferisce l'esistenza di un Casale Syponti ${ }^{20}$, definizione che torna in un rogito del $1180^{21}$. Il toponimo fa sorgere molti dubbi. Può parlarsi di una ruralizzazione della città? Questa, dopo il presunto evento traumatico del 1155 , viene percepita piuttosto come un casale dal redattore del documento? O invece esiste un effettivo Casale Syponti, quale entità demica autonoma, sito in ubicazione diversa dalla città? ${ }^{22}$ La tesi di una ruralizzazione appare piuttosto plausibile se si considera che in un documento del 1190 si attesta l'esistenza di alcune terre prive di costruzioni all'interno dell'antico perimetro della civitate veteris Siponti insieme ad una chiesa intitolata a Sant'Andrea ${ }^{23}$. Un altro documento del 1188 invece cita una civitate nova Siponti ${ }^{24}$. La questione si complica notevolmente. Si parla del casale ora assurto nuovamente a città? Le fonti tacciano al riguardo. Eppure pare davvero poco probabile. Tuttavia, nel 1204 Siponto viene nuovamente qualificata come civitas, mentre i documenti testimoniano dal 1212 la ripresa delle normali funzioni cittadine ${ }^{25}$.

De Troia ipotizza la nascita di un aggregato demico, definito «casale» in un luogo diverso da Siponto, identificabile con la strata magna che conduce al santuario di San Michele. Un'ipotesi che si scontra con una cronologia forse fin troppo precoce. Tesi che non esclude però la possibilità di una serie di nuclei abitativi sparsi lungo la strada. Sappiamo dell'esistenza nei pressi dell'asse viario del monastero di S. Benedetto già dal 1201. Nel 1180 viene poi segnalata iuxta stratam magnam la casa di Benesmiro di Siponto,

19 Ioannis Cinnami Epitome rerum ab Ioanne et Alexio Comnenis gestarum, Meineke, A. (ed.). Corpus Scriptorum Historiae Byzantinae. Bonn: Weber, 1836.

20 Martin, Jean Marie. "Codice Diplomatico Pugliese», n. 47, 1175, pp. 138-139; n. 49, 1185, pp. 141-143; n. 51, 1182, pp. 145-146. Cfr. anche Regesto di San Leonardo di Siponto, doc. 89, 1180, pp. 56-57; Martin, "La città di Siponto», pp. 15-32, in particolare p. 31; De Troia, Dalla distruzione, pp. 21-22.

${ }_{21}$ Regesto di San Leonardo di Siponto, doc. n. 41, 1155, pp. 27-28; cfr. Martin, "La città di Siponto», p. 31 .

22 Martin, «Codice Diplomatico Pugliese», n. 49, 1185, pp. 141-143; n. 51, 1182, pp. 145-146; Martin, "La città di Siponto», pp. 21-22.

23 Regesto di San Leonardo di Siponto, doc. n. 104, 1193, pp. 65-66; n. 107, 1196, pp. 67-68; n. 109, 1196, pp. 68-69.

24 Martin, «Codice Diplomatico Pugliese», n. 57, 1188, pp. 157-158.

25 Quaternus de excadenciis et revocatis Capitinatae de mandato imperialis maiestatis Friderici secundi, Amelli, A. (ed). Montecassino: San Paolo, 1903, pp. 48-50; De Troia, Giuseppe. Foggia e la Capitanata nel Quaternus excadenciarum di Federico II di Svevia. Fasano: Schena, 1994, p. 89. 
giustiziere dell'Honor Sancti Angeli ${ }^{26}$. La presenza di un nutrito numero di case presso la strata magna è confermata in un'epistola del 3 novembre 1277, indirizzata da re Carlo al Giustiziere Guglielmo de Sactais. La lettera menziona gli accordi intercorrenti con il mastro Giordano da Monte Sant'Angelo volti a rifunzionalizzare le pietre con cui sono costruite le case, a quel tempo in abbandono, collocate a nord della strata, la cosiddetta Ruga de Comite. Un numero di case certamente consistente, poiché i proprietari inviano al re una petizione nell'aprile del 1278 per salvaguardare i propri diritti. Il re nomina una commissione ad hoc: di essa fanno parte il prefato Giustiziere, il giudice Benedetto, l'architetto Pietro d'Angicourt ed alcuni probiviri della città, che sono incaricati di fare una stima del valore delle pietre. Sicché i proprietari devono rifondere le maggiori spese sofferte per il reperimento di materiale alternativo, salvo incameramento da parte dell'ente locale ${ }^{27}$. L'ingente spesa richiesta all'Università ne dimostra il numero nutrito.

Seguendo l'ipotesi di De Troia, alla distruzione di Siponto, probabilmente limitata allo smantellamento delle sole mura, si accompagna l'aggregarsi di una parte della popolazione in un casale. Una suggestione davvero forte, corroborata dal Breve chronicon de rebus siculis, che in un passo problematico attribuisce a Guglielmo I (1120-1166 d.C.) la distruzione di Siponto insieme alle città di Bari e Trani. La fonte afferma: Dominus vero rex (...). Destruxit etiam Sipontum et habitatores ipsius collocari fecit in eo loco, in quo modo est Manfredonia ${ }^{28}$. Tale informazione rende più credibile l'ipotesi di una dispersione degli abitanti, che viene causata da una rappresaglia contro Siponto, poiché finalizzata a punire la città simpatizzante per i bizantini. La situazione incorsa ai sipontini non appare troppo chiara: esistono due differenti lezioni del testo del De Rebus Siculis, che narrano le conseguenze della spedizione militare. Il codice VIII C 9 della Biblioteca Nazionale di Napoli attesta che i Baresi ed i Sipontini vivono esuli per 11 anni, fino alla morte di Guglielmo I deceduto nel 1166. Diversamente il codice Ottoboniano Latino 2940 riporta che i Tranesi e i Sipontini restano esuli per 40 anni, fino al 1195, anno della morte di Enrico VI,

26 Palumbo, Pier Fausto. «Honor Montis Sancti Angeli». Archivio Storico Pugliese, 1953, vol. IV, pp. 304-370.

27 De Troia, Dalla distruzione, pp. 32; 108; Vendola, Domenico. Documenti tratti dai registri vaticani da Bonifacio VIII a Clemente V. Trani: Vecchi, 1963, doc. n. 28, p. 27; vedi anche doc. n. 644, Reg. Ang., vol. XVIII, p. 320; doc. n. 543, Reg. Ang., Vol. XVIII, pp. 254-255; doc. n. 604, Reg. Ang., vol. XVIII, p. 292.

Breve chronicon De Rebus Siculis, Delle Donne, Fulvio (ed.). Roma: Istituto Storico Italiano per il Medioevo, 2017, pp. 7-8; Delle Donne, Fulvio. "Tradizioni testuali e (ri)scrittura della storia. Il caso del Breve chronicon de rebus Siculis». Zeitschrift Quellen und Forschungen aus italienischen Archiven und Bibliotheken Band, 2016, vol. XCVI, pp. 178-179. Il testo del De Rebus Siculis è conservato in 3 manoscritti: il manoscritto N, ovvero il codice VIII C 9, della Biblioteca Nazionale di Napoli, riporta il testo alle cc. 101r-107v; il manoscritto V, ossia l'Ottoboniano Latino 2940, presso la Biblioteca Apostolica Vaticana, lo riferisce alle cc. 42v-48v; il manoscritto $\mathrm{V}^{2}$, il Vaticano Latino 7145, sempre presso la Biblioteca Apostolica Vaticana, invece lo restituisce alle cc. $1 \mathrm{r}-13 \mathrm{v}$. Di seguito si riportano le due differenti lezioni. Il passo controverso è riportato nel codice VIII C 9 al f. 101v, laddove esordisce: Destruxit etiam Sipontum et habitatores ipsius collocari fecit in eo loco, qui Maletangium dicebatur, in quo modo est Manfredonia. Diversamente l'Ottoboniano Latino 2940 al f. 42v riporta: Destruxit etiam Sipontum et habitatores ipsius collocari fecit in eo loco, in quo modo est Manfredonia. 
allorché sono richiamati in città da Costanza. Prescindendo dai problemi ingenerati dalle diverse lezioni dei manoscritti, occorre considerare che un qualsivoglia evento traumatico subito dalla città ha funzionato da catalizzatore per una ruralizzazione dell'abitato, forse già in atto, facendolo percepire come diruto. Il De Rebus Siculis sembra così confermare il 1156 come termine ante quem per il deterioramento del tessuto urbano.

Rimangono aperte ulteriori questioni. Alla luce delle fonti può apparire ragionevole una doppia distruzione di Siponto, avvenuta a circa un anno? Si può poi presupporre che immediatamente dopo «nasca» il cosiddetto Casale Syponti? Innanzitutto, appare poco plausibile che gli abitanti di Siponto si aggreghino subito dopo il trauma nel casale, laddove mantengono simpatie filobizantine. Nondimeno, pare fin troppo strano che le simpatie degli abitanti del casale non abbiano offerto alcun ulteriore pretesto di rappresaglia. Al contrario, si dovrebbe ammettere che Siponto è oggetto di una seconda ed insensata spedizione punitiva. Pare più razionale parlare di un'unica rappresaglia, che incide un tessuto urbano già in deterioramento per i fenomeni ipotizzati da Martin. Al contempo, bisogna dubitare dell'effettiva esistenza del cosiddetto "casale» già dall'estate del 1156. Altrimenti, si è costretti a sostenere un'improbabile ipotesi: l'abitato è del tutto ignorato o persino sconosciuto al $\mathrm{re}^{29}$. Pare altrettanto poco probabile la sopravvivenza di una nutrita enclave apertamente filobizantina anche a seguito della resa di Benevento ${ }^{30}$. E se si considera che il documento costituisce un unicum, lo si deve necessariamente qualificare come il "canto del cigno» della fedeltà dei locali al basileus. Ogni simpatia verso i romano orientali è destinata a spegnersi poco dopo questa data.

I toponimi vetus Siponto e casale Syponti alla luce dei dati raccolti non possono che indicare rispettivamente le due aree più importanti del territorio cittadino: il sito dell'insula episcopalis ed il suo porto dotato di strutture difensive, quale l'arx citata da Ughelli, che in quel periodo la fa sembrare un'entità autonoma rispetto al centro spirituale della città ${ }^{31}$. L'assenza di ulteriori citazioni del casale dopo il 1198 lascia pensare che tale struttura venga prontamente riassorbita nel tessuto urbano, il cui cuore rimane la cattedrale. Nondimeno, si ritiene poco probabile che tale casale scompaia dalle fonti perché abbandonato ${ }^{32}$.

29 De Troia, Dalla distruzione, pp. 60-61; 69-70; Violante, «Da Siponto a Manfredonia», pp. 1617

30 Gay, Giulio. L'Italia meridionale nell'impero bizantino. Bologna: Forni, 1978, p. 334; TABACCO, Giovanni. «Il potere politico nel Mezzogiorno d'Italia dalla conquista normanna alla dominazione aragonese». In Il Mezzogiorno medievale nella storiografia del secondo dopoguerra: risultati e prospettive. Atti del IV Convegno dell'Associazione dei Medioevalisti Italiani, Università di Calabria, 1982. Cosenza: Rubbettino, 1985, pp. 65-111.

31 Violante, «Da Siponto a Manfredonia», p. 19; SAnfilippo, Mario. «Le città pugliesi dall'xi al xV secolo: continuità e persistenze negli insediamenti difensivi». In SANFILIPpo, Mario (ed.). Medioevo e città nel regno di Sicilia e nell'Italia comunale. Messina: Sicania, 1991, pp. 37-53.

32 Violante, «Da Siponto a Manfredonia», pp. 18-19; De Troia, Dalla distruzione, p. 37. 
Gli eventi che intercorrono fra il 1155 ed il 1156 sembrano avere poche implicazioni sull'infrastruttura portuale e sulle sue attività. Da questo porto Alessandro III in una data fra il 1176 ed il 1177 si imbarca per Venezia. Ciò dimostra l'esistenza di navi con tratta medio-lunga, che partono o fanno spola a Siponto.

Dal Quaternus de excadenciis abbiamo conoscenza che poco più tardi, ovvero nel 1205, le mura di Siponto sono fornite di un barbacane e di una grande porta protetta da una torre; la città invece è dotata di un fundicum (un magazzino pubblico) ${ }^{33}$. Nella prima metà del XıII secolo si segnala un fervore delle attività commerciali del porto ${ }^{34}$, che spinge nel 1231 a dotare Siponto di uno Statutum custodibus fundicorum, con cui si fissano i diritti di fondaco e quelli di dogana per le merci indirizzate extra regnum ${ }^{35}$.

Ma di quale porto si tratta? Sappiamo che in quegli anni il porto di Siponto, sito nella zona ora denominata Orto delle brecce ${ }^{36}$, diviene progressivamente inutilizzabile per il fenomeno di insabbiamento dovuto alla stratificazione dei sedimenti dei fiumi Ofanto e Candelaro ${ }^{37}$. I fattori ecologici accorsi durante il sec. XIII, come gli spostamenti della foce del Bradano e dell'Ofanto dovuti in parte al dissodamento dei terreni sottratti alle acque dall'uomo per dedicarli alla coltura, concorrono alla ridefinizione della $\operatorname{costa}^{38}$. L'impatto delle operazioni umane sull'equilibrio idrogeologico del Tavoliere ha avuto ripercussioni sul territorio sipontino, che diviene soggetto ad impaludamento.

33 Quaternus de excadenciis et revocatis Capitinatae, pp. 48-58; MARTIN, "La città di Siponto", p. 31. 34 De Troia, Dalla distruzione, pp. 37-38; 47; 53.

35 Ognissanti, «Il porto di Siponto», pp. 9-51.

36 In antichità Siponto funge da propaggine portuale per la vicina città di Arpi a cui è forse sottomessa, fino a che non viene rifondata nel 194 d.C. come colonia romana (cfr. Liv., Ab urbe condita, XXXIV, 45; XXXIX, 23). L'innalzamento a colonia della città è perciò agevolato dalla posizione logistica in cui si ubica, giacché si stende alle pendici del Gargano e nei pressi di un'insenatura, lunga $2500 \mathrm{~m}$ e larga tra i 600 ed i $700 \mathrm{~m}$. Questa viene parzialmente chiusa da due braccia costituite in parte da sedimenti marini, in parte da sedimenti fluviali che fungono da approdo naturale e la rendono un porto alquanto funzionale. La vitalità dell'infrastruttura portuale è meglio significata dal fatto che la città ha accolto un collegio di negotiatores. Cfr. Martin, "La città di Siponto», pp. 16-18; per l'istituzione dei negotiatores si veda: Cass., Variae, MGH, AA, XII, II, 38.

37 Martin, Jean Marie e Noyé, Ghislaine. «Les campagnes de l'Italie méridionale byzantine $\left(\mathrm{x}^{\mathrm{e}}-\mathrm{XI}^{\mathrm{e}}\right.$ siècles)». Mélanges de l'École Française de Rome - MoyenAge, 1989, vol. CI, pp. 558-596; DeLANo SMith, Chatrine. "Tipi di insediamenti nella zona costiera di Foggia». In Civiltà preistoriche e protostoriche della Daunia. Firenze: Istituto Italiano di Preistoria e Protostoria, 1975, pp. 5-15; Delano Smith, Chatrine. Daunia vetus. Napoli: Tipografia Laurenziana, 1978, pp. 72-78.

38 Martin, Jean Marie. La vita quotidiana nell'Italia Meridionale ai tempi dei Normanni. Torino: Bur, 2018; Neboit, René. «Les basses terrasses alluviales, témoins de l'occupation des sols. Italie méridionale, Sicile». In Noyé, Ghislaine (ed.). Structures de l'habitat et occupation du sol dans les pays méditerranéens: les méthodes et l'apport de l'archéologie extensive. Actes de la rencontre organisée par l'École Française de Rome, Paris, 12-15 novembre 1984. Rome-Madrid: École Française de Rome-Casa de Velázquez, 1988, pp. 401405; Sснміеdт, Giulio. "Contributo della fotografia aerea alla ricostruzione della antica laguna compresa tra Siponto e Salapia». Archivio Storico Pugliese, 1973, vol. XXVI, pp. 159-172. 
Nonostante ciò le fonti attestano una notevole frequentazione del molo. Nel 1235 Federico II parte da Siponto per dirigersi via mare ad Aquileia ${ }^{39}$. Nel 1236 ivi sbarcano le due galere con Enrico re di Germania ${ }^{40}$. Sappiamo pure che Siponto è un porto militare data la presenza nel 1236 di un comitus a comando di una nave da guerra e di un prothontinus, un comandante militare posto a difesa del litorale ${ }^{41}$. Nel 1240 giungono a Siponto ben 24 galere veneziane, dopo aver saccheggiato alcune città del Gargano. Qui catturano una nave con mille uomini a bordo. Lo stivaggio delle galee veneziane e la presenza di una nave capace di portare così tanti passeggeri fanno pensare ad una infrastruttura atta a sopportare imbarcazioni di alto cabotaggio, sia da carico, che da guerra. E tutto questo sembra avvenire durante il suo progressivo insabbiamento. L'ampia capacità del porto è confermata dal fatto che accoglie nel 1252 il re di Sicilia Corrado IV insieme ad un esercito di tedeschi portato da navi veneziane ${ }^{42}$. Nel 1255 sbarca a Siponto l'esercito pontificio capitanato da Bertoldo di Hohenburg, proclamato dal papa legittimo erede del trono siciliano, e qui si consuma un primo scontro con Manfredi ${ }^{43}$.

Il 1257 vede altresì la concessione a Genovesi e Veneziani da parte di Manfredi di suoli per l'edificazione di fondachi nei pressi del porto insieme a contributi pro logiis faciendis ed alla facoltà di nominare consoli ${ }^{44}$.

Un porto così attivo potrebbe essere identificato con un'altra infrastruttura: quella di Cala dello Spontone. Un porto suburbano, sito a circa tre $\mathrm{km}$, dotato di alcune strutture sanitarie come l'ospedale di S. Lazzaro (il lazzaretto), posto ad occidente della cala, di cui si hanno informazioni sin dal 1221. I documenti in nostro possesso non ci permettono però di distinguere se quel porto così vitale nelle prime decadi del sec. XIII possa essere effettivamente identificato con quello di Cala dello Spontone.

Laddove vi è un porto, vi sono dei fondaci e gli addetti alle attività portuali sicuramente preferiscono vivere in luoghi limitrofi alla propria occupazione e non certo a tre $\mathrm{km}$ dal posto di lavoro. Ciò offre una ragionevole presunzione: le abitazioni proliferate attorno al porto costituiscono parte del nucleo da cui sorgerà Manfredonia. Emerge l'importante ruolo dell'infrastruttura come aggregatore demico, poiché capace di stimolare l'antropizzazione del paesaggio suburbano di Siponto.

La nascita di Manfredonia poi deve essere contestualizzata nel quadro geografico, sociale ed economico della Capitanata dei secc. XII-XIII. Il presunto intervento regio del 1263 sembra inserirsi in un più ampio progetto di revisione dei nuclei difensivi della costa del Regno ${ }^{45}$. Al contempo, si deve sottolineare come l'emergenza sanitaria dovuta all'insalubrità dell'aria della zona prossima a Siponto abbia giocato un ruolo fondamentale nella

39 Historia diplomatica Friderici Secundi, Huillard-Bréholles, Jean-Louis-Alphonse (ed.). Paris: Plon, 1852-1861, I/1, p. 905.

$40 \quad$ Historia diplomatica Friderici Secundi, I/1, p. 903.

41 Regesto di San Leonardo di Siponto, doc. n. 191; Martin, «La città di Siponto», p. 29.

42 De Troia, Dalla distruzione, p. 57.

43 Ivi, p. 59.

44 Ivi, p. 60; Abulafia, David. Le due Italie. Relazioni commerciali tra il Regno normanno di Sicilia e i comuni dell'Italia settentrionale. Napoli: Guida Editori, 1991, pp. 8-12; 126-129.

45 Johannes Victoriensis. Chronicon Carinthiae, Böhmer, J. F. (ed.). Fontes Rerum Germanicarum. Stuttgart: Verlag, 1843-1868, I, p. 288. 
progettazione della nuova città. Dal Datum Orte emerge un preciso riferimento a tale problematica: propter ipsius loci intemperiem, et imminentem ibi corruptionem aeris, che esige una migrazione dei sipontini secondo un «itinerario atipico ed originale» ${ }^{46}$, poiché nella nuova sede habetur aeris puritas ${ }^{47}$. Villani riferisce che l'insalubrità del clima stimola la nuova fondazione ${ }^{48}$. Precisa il concorso di fattori di natura economico-commerciale, come la buona posizione logistica. L'infrastruttura si dimostra fondamentale per una prospera vita cittadina e funge da attrattore per i soggetti che cercano buoni affari.

Le ragioni sanitarie e le esigenze di natura economica vengono pure menzionate nella cronaca del sec. XIII di Ricordano Malispini ${ }^{49}$. Cosa che non stupisce, poiché quest'ultima presenta fequenti similitudini con la cronaca del Villani ${ }^{50}$. A Malispini poi sembra aver attinto Benvenuto da Imola. Il quale però indugia su una più ampia descrizione dell'infrastruttura marittima, allorché afferma l'esistenza di un portum cum muro amplissimum intra mare, quae molum vocant ${ }^{51}$. L'annotazione dimostra che questi ha presente un'altra fonte, ad oggi sconosciuta ${ }^{52}$.

Il ruolo dell'infrastruttura portuale nella progettazione e nella sopravvivenza del nuovo aggregato demico diviene imprescindibile: le ragioni della mercatura fanno così passare in secondo piano quelle sanitarie. Emerge la prepotente necessità di uno snodo commerciale marittimo, indispensabile allo smaltimento delle eccedenze agricole della Capitanata, costituendo un cosiddetto "agriporto" ${ }^{53}$.

Sin dal 1235 sorge l'esigenza di ridefinire le linee portuali del litorale pugliese e si sceglie di indirizzare entro percorsi prestabiliti tutto il surplus agricolo e la produzione di

46 Iorio, Raffaele. «Siponto, Canne». In Musca, Giosuè (ed.). Itinerari e centri urbani nel Mezzogiorno normanno-svevo. Atti delle decime giornate normanno-sveve. Bari, 21-24 ottobre 1991. Bari: Dedalo, 1993 , p. 423

47 Datum Orte, cit. in Serricchio, «Manfredi», p. 508.

48 Giovanni Villani. Nuova cronica, Porta, Giuseppe (ed.). Parma: Ugo Guanda Editore, 19901991, libro VII, cap. XLVI.

49 Ricordano Malispini. Istoria fiorentina, Muratori, Ludovico A. (ed.). Rerum Italicarum Scriptores, VII. Milano, 1726, cc. 881-1028, c. 978 D, cap. 98.

50 La paternità dell'opera di Malispini non è pacifica. Prima di tutto non esiste un documento che affermi con certezza l'esistenza di un Ricordano entro la famiglia Malispini, tanto che si tende ad additare l'opera ad un anonimo che, partendo dalla storia fiorentina di Giovanni Villani, vi interviene con non troppe aggiunte. Per la recente bibliografia sulla questione cfr. MAstroddi, Laura. "Contributo al testo critico della Storia fiorentina di Ricordano Malispini». Bullettino dell'Istituto Storico Italiano per il Medioevo, 2000-2001, vol. CIII, pp. 239-293; Porta, Giuseppe. «Le varianti redazionali come strumento di verifica dell'autenticità dei testi: Villani e Malispini». In Guida, Saverio e Latella, Fortunata (edd.). La filologia romanza e $i$ codici. Atti del Convegno della società italiana di filologia romanza, Messina 12-19 dicembre 1991. Messina: Signa, 1993, pp. 481-487; CRISTIANI, Emilio. "I più recenti orientamenti sulla "Storia fiorentina" malispiniana". Bolletino Storico Pisano, 2001, vol. LXX, pp. 305-310.

51 Benvenuti de Rambaldis de Imola Comentum super Dantis Aldigherij Comoediam, Lacaita, James Philip (ed.). Firenze, 1887, III, p. 102.

52 IORIO, «Siponto», p. 400.

53 Violante, "Da Siponto a Manfredonia», p. 21; Licinio, Raffaele. Masserie medievali. Masserie, massari e carestie da Federico II alla Dogana delle pecore. Bari: Adoa, 1989, pp. 123-124; Rivera Magos, Victor. "La chiave de tutta la Puglia. Presenze straniere, attività commerciali e interessi mediterranei a Manfredonia, 'agriporto' di Capitanata (secoli XIII-Xvi)». In Licinio, Raffaele (ed.). Storia di Manfredonia. Il Medioevo. Bari: Edipuglia, 2008, pp. 63-99. 
grano del regio demanio. Nel 1239 segue una disposizione che obbliga tam incole regni quam advene, undecumque sint, preter venetos ad essere vincolati solo a predeterminati porti, laddove è possibile esportare sia il vettovagliamento, che i capi di bestiame. Porti come quelli di San Cataldo e Torreamare per la Terra di Bari e di Rivoli per la Capitanata. Tale predisposizione non tiene conto delle realtà territoriali e porta l'esperimento al fallimento, come dimostra la scomparsa a metà del Duecento del porto di Rivoli ${ }^{54}$.

La Capitanata costituisce una sorta di «laboratorio» economico, che deve garantire buoni introiti al Fisco regio attraverso l'applicazione dei diritti sui generi oggetto di monopolio e sulle merci in uscita. A queste si aggiungono imposte quali l'anchoraticum, l'arboraticum, lo scalaticum e lo ius dohane. Quest'ultima tassa incide fortemente sul valore delle transazioni, tanto che compare negli atti di vendita con la formula: jure dohanne dumtaxat excepto $0^{55}$.

A metà del XIII sec., allorché si verifica l'interramento del porto di Siponto, viene a mancare un importante termine di scambio marittimo per l'economia della Capitanata. La progressiva inutilizzabilità dello scalo crea l'urgenza di un nuovo snodo marittimo destinato al commercio non del solo surplus agricolo, ma anche di beni di varia natura come il sale ed i cereali. Una funzione che si inserisce nella trama dei rapporti internazionali intessuti dal Regno di Sicilia. Allorquando si offre spazio ai mercanti stranieri, specie genovesi e veneziani, che sono sempre più presenti nelle città costiere di Puglia ${ }^{56}$.

La vocazione commerciale della nuova città è fortemente stigmatizzata dal privilegio: victualia omnia iustitiariatus Capitanate quaeper mare concessimus extrahenda per quoscumque de iurisdictione ipsa extrahi debeant, et liceant de portu Civitatis ejusdem, et non alio, tantummodo extrahantur. Adjicimus (... ${ }^{57}$. Al fine di garantire un buon numero

54 Circa le disposizioni sul sistema portuale, meglio conosciute sotto il nome di Edictum super portubus, cfr. Il registro della cancelleria di Federico II del 1239-1240, Carbonetti Venditelli, Cristina (ed.). Roma: Istituto Storico Italiano per il Medio Evo, 2002, I, n. 29, 5 ottobre 1239, pp. 22-27; n. 30, 5 ottobre 1239, pp. 27-31. Per le caratteristiche del «sistema» federiciano e circa il «laboratorio» di Capitanata, cfr. Licinio, Raffaele. "I poteri territoriali: re, signori, vescovi e città». In Salvemini, Biagio e Massafra, Angelo (edd.). Storia della Puglia. Dalle origini al Seicento. Roma-Bari: Laterza, 2005, pp. 130-149; Licinio, Raffaele. «La normativa sul sistema masseriale». In Musca, Giosuè (ed.). Le eredità normanno-sveve nell'età angioina. Persistenze e mutamenti nel Mezzogiorno. Atti delle quindicesime giornate normanno-sveve. Bari, 22-25 ottobre 2002. Bari: Dedalo, 2004, pp. 197-218; Del Treppo, Mario. «Prospettive mediterranee della politica economica di Federico II». In Esch, Arnold e Kamp, Norbert (edd.). Friedrich II. Tagung des Deutschen Historischen Instituts in Rom im Gedenkjahr. Tübingen: Bibliothek des Deutschen Historischen Instituts in Rom, 1996, pp. 316-338; Del Treppo, Mario. «Federico II e il Mediterraneo». Studi Storici, 1996, vol. XXXVII, pp. 373-390, in particolare pp. 382-390.

55 Barile, Nicola Lorenzo. "Merci e mercati della Capitanata medievale: la testimonianza delle "pratiche di mercatura" ". In Gravina, Armando (ed.). Atti del $33^{\circ}$ Convegno Nazionale sulla Preistoria, Protostoria, Storia della Daunia. San Severo: Centro Grafico S.r.l., 2013, p. 181; Bianchini, Ludovico. Storia delle finanze del Regno delle due Sicilie, De Rosa, Luigi (ed.). Napoli: Edizioni Scientifiche Italiane, 1971, p. 119; Cassandro, Giovanni. "I porti pugliesi nel medioevo». In Cassandro, Giovanni (ed.). Saggi di storia del diritto commerciale. Napoli: Edizioni Scientifiche Italiane, 1982, pp. 355-394.

56 Del Treppo, Mario. «Stranieri nel Regno di Napoli. Le élites finanziarie e la strutturazione dello spazio economico e politico». In Rossetti, Gabriella (ed.). Dentro la città. Stranieri e realtà urbane nell'Europa dei secoli XI-XV. Napoli: Liguori, 1989, pp. 179-233.

57 Datum Orte, cit. in SerricCHIO, «Manfredi», p. 508. 
di transazioni a quello scalo marittimo ed entrate fisse per il Regno, si dispone che le merci prodotte dall'entroterra della Capitanata si indirizzino per privilegio regio tutte a quel porto. L'infrastruttura viene progettata fin dall'inizio per sostenere alti volumi di traffico di merci, tanto che Salimbene de Adam sottolinea come Manfredonia habet optimum portum ${ }^{58}$. Malispini poi, riprendendo letteralmente Villani, entusiasticamente lo definisce il miglior porto, che sia da Vinegia a Brundizio ${ }^{59}$.

La nuova fondazione assume maggior significato entro il ripensamento da parte dell'autorità regia dell'assetto economico dell'Alto Tavoliere e, come tale, viene percepita dai cronisti che sono colpiti dal dispendio di risorse per ottimizzare la funzionalità del suo porto. La concessione di privilegi ed esenzioni mira alla costruzione e all'ampliamento di una classe mercatile entro una città, che l'effettiva posizione e le disposizioni regie rendono strategica. Il porto si dimostra pure utile ad aprire canali commerciali con l'Oriente bizantino. Le seconde nozze di Manfredi con Elena Comneno ${ }^{60}$, figlia di Michele II despota d'Epiro, creano nel 1259 un ponte diretto fra le due sponde dell'Adriatico e stimolano una serie di scambi di natura sia materiale, che immateriale. Dopotutto Elena porta in dote Corfú, i diritti su Durazzo ed il suo agro, Valona, Berati e altre città d'Albania; apre poi una linea di collegamenti con la costa albanese in cui il porto di Manfredonia può inserirsi agevolmente ${ }^{61}$. La politica di riavvicinamento con l'oriente cristiano, che Manfredi realizza a mezzo matrimoniale, segue l'esempio del padre Federico, il quale ha dato in sposa la sorella Costanza a Giovanni III Ducas Vatatze.

Il ruolo svolto da questo porto nel garantire entrate costanti al regno giustifica pure la premura con cui i sovrani angioini, Carlo I e Carlo II, si interessano al completamento e al potenziamento del nucleo urbano ${ }^{62}$. Un volume di commerci piuttosto rilevante che è anche attestato dai Registri della Cancelleria Angioina ${ }^{63}$ tra il 1269 ed il 1290.

Un documento del 1279, rogato apud Sypontum Novellum, ci informa sulla presenza di fosse granarie ubicante in città di proprietà dell'Università sulle quali è esercitato un controllo da parte di magistrati appositi e dai padri Domenicani, a cui si aggiungono

58 Salimbene de Adam. Cronica, Scalia, Giuseppe (ed.). Roma-Bari, Laterza, 1966, II, p. 685.

59 Ricordano Malispini, Istoria fiorentina, cc. 881-1028, c. 978 D.

60 http://www.treccani.it/enciclopedia/elena-di-epiro-regina-di-sicilia_(Dizionario-Biografico)/

${ }_{61}$ Sarcinelli, Giuseppe. "La collezione numismatica del Comune di Manfredonia». In MazzeI, Marina (ed.). Siponto antica. Foggia: Grenzi Editore, 1999, p. 82; Palumbo, Pier Fausto. Contributi alla storia dell'età di Manfredi. Roma: Edizioni del Lavoro, 1959, p. 104.

62 Carabellese, Francesco. Le relazioni commerciali fra la Puglia e la Repubblica di Venezia dal secolo X al XV. Bologna: Forni, 1991; Pryor, John H. «Foreign policy and economic policy: the Angevins of Sicily and the economic decline of Southern Italy». In Frappell, L.O. (ed.). Principalities, powers and estates studies in medieval and early modern government and society. Adelaide: Adelaide University Union Press, 1980, pp. 43-55; Prencipe, Domenico. "L'economia di Manfredonia nel periodo protoangioino». Bollettino del Nuovo Centro di Documentazione Storica, 2001-2004, vol. VI, pp. 201-220; Poleggi, Ennio. "La costruzione della città portuale, un nuovo tema di storia». In Poleggi, Ennio (ed.). Città portuali del Mediterraneo. Storia e archeologia. Atti del Convegno Internazionale Città portuali del Mediterraneo, Genova, 1985. Genova: Sagep Editrice, 1989, pp. 7-10.

63 Ognissanti, «Il porto di Siponto», p. 15, nota 40. 
quelle regie presso il porto. Ivi si rivolgono i Veneziani e i Fiorentini, che dal 1288 al 1301 acquistano 3.087 salme di frumento e 868 salme di orzo $^{64}$.

Sappiamo che nel 1299 i Bardi, i quali hanno finanziato gli Angiò, fanno partire da Manfredonia ben 31.290 salme di grano, il cui prezzo viene scorporato dal debito contratto dalla casa regnante. Sotto Roberto d'Angiò e con più precisione nel 1314 si concede ai mercanti fiorentini Tommaso, Giotto e Donato Perruzzi, a Giovanni Bartoli ed a Geri Stefani il privilegio di estrarre grano da quel porto ${ }^{65}$. Firenze è dunque il partner principale delle transazioni commerciali per quanto concerne il vettovagliamento.

La forte presenza dei fiorentini a Manfredonia è testimoniata da Pegolotti, membro della compagnia dei Bardi e redattore nel 1340 di una pratica della mercatura ${ }^{66}$. Costui, sebbene soggiorna a Barletta, offre importanti notizie sul porto di Manfredonia. Questi consiglia di acquistare a Manfredonia oltre al grano in ragione della convenienza della «salma» (la misura applicata) anche l'olio, che qui sembra avere costi minori a causa delle ridotte spese richieste per l'imbarco ${ }^{67}$.

Gli Angiò introducono pure la viticultura nel 1280, ponendo le vigne sotto la sovrintendenza di Ursone Pastore, magister massariarum di Capitanata ${ }^{68}$. Meno informazioni invece ci giungono sull'olivicoltura.

Un porto che si inserisce nel complesso sistema rappresentato dalla Carta Portolano, che fotografa la gerarchia portuale della Puglia alla fine del secolo XIII. Nel 1301 Carlo II predispone un programma di disciplinamento fiscale per Manfredonia e ci informa circa tutti i beni che vi si possono rinvenire: victualibus, leguminibus, vino, oleo, caseo ricocto, carnibus salatis cuiuscumque generis, piscibus recentibus et salatis, fructibus viridibus et siccis, lana ovium, bombice, et sete filate, cannape, lino, ferro, aczario, ere et omni genere metallorum, cera, melle, pice, et omnis peciaria, pellibus et coriis, pellementaria ${ }^{69}$. Questo porto costituisce un emporio ben fornito, mentre l'entroterra pare capace di offrire una buona varietà di produzioni. L'offerta è tale da soddisfare non solo le esigenze alimentari del territorio, ma produce anche del surplus che lo snodo agevola a smaltire. I dazi applicati a Manfredonia sono costruiti sul precedente regolamento regio emanato per Barletta nel 1297 da Carlo II, laddove sono incardinati il Maestro Portolano di Puglia

64 Ognissanti, «Il porto di Siponto", p. 17: le salme corrispondono all'incirca a 4.538 quintali di frumento e 1.276 quintali di orzo.

65 Ivi, pp. 17-18.

66 BARILE, «Merci e mercati», pp. 179-181.

67 Pegolotti riferisce: la «misura di Manfredonia, chè quella di Barletta si è maggiore che quella di Manfredonia $2 \frac{1}{2}$ per 100 , ma perché si carica più in Manfredonia che a Barletta...» cfr. FrANCESCO BALducci Pegolotti. La pratica della mercatura, Evans, Allan (ed.). Cambridge Mass: Medieval Academy of America, 2012, p. 116; Giagnacovo, Maria. Appunti di metrologia mercantile genovese: Un contributo della documentazione aziendale Datini. Firenze: Firenze University Press, 2014, p. 126.

68 Licinio, Masserie, pp. 159; 188; Morelli, Serena. "Una fonte inedita per la storia del Regno di Napoli in età angioina: le carte di Leon Cadier». Rassegna degli Archivi di Stato, 1997, voll. II-III, pp. 471474.

69 ASCM, Carte antiche, 1.4, cc. 1-8, pp. 3-4; Violante, Francesco. «Organizzazione del territorio e strutture produttive tra XI e Xvi secolo». In Licinio, Raffaele (ed.). Storia di Manfredonia. Il Medioevo. Bari: Edipuglia, 2008, pp. 101-123. 
IL PORTO E LA STRADA. SPUNTI DI RIFLESSIONE SULLA TRADIZIONE CONCERNENTE LA FONDAZIONE DI MANFREDONIA ANTONIO PIO DI COSMO

e l'ufficio dei Maestri Razionali; autorità a cui il porto di Manfredonia è sottoposto ${ }^{70}$. Dalla comparazione del testo del privilegio di Barletta con quello di Manfredonia, si evidenzia una certa dipendenza del formulario adoperato per l'ultimo documento. E non può essere altrimenti. I due territori comunali a quella data sono confinanti e si pongono, a mezzo di una regolamentazione omogenea della pretesa fiscale, in dialogo tra loro. La disciplina fiscale comune soddisfa le esigenze di un'economia locale agricola, dipendente nei territori della Capitanata, nell'area dell'Ofanto ed in quella antistante le Murge dalle masserie regie. Si raffronta così una strategia che risponde alle necessità del Fisco e vuole ottimizzare le entrate rispetto alla domanda esterna al Regno. Il regolamento, costituito da ben 43 capitula $^{71}$, poi deve tenere conto delle peculiarità della città e del territorio che va a normare. Il testo lascia emergere una forte suggestione: sembra che Manfredonia venga fondata per fungere da "porto agricolo» per il Tavoliere, «costruita intorno ad esso e per esso" ${ }^{72}$. La città poi sembra reggersi su quei dazi concessi. A ragione la classe dirigente locale li custodisce gelosamente come "privilegio di classe» e cerca di perpetuarli, richiedendone continuamente la conferma alla Corona. Nonostante le peculiarità, si può affermare che nel sec. xIV Manfredonia e Barletta appaiono come parte integrante di un unico sistema economico.

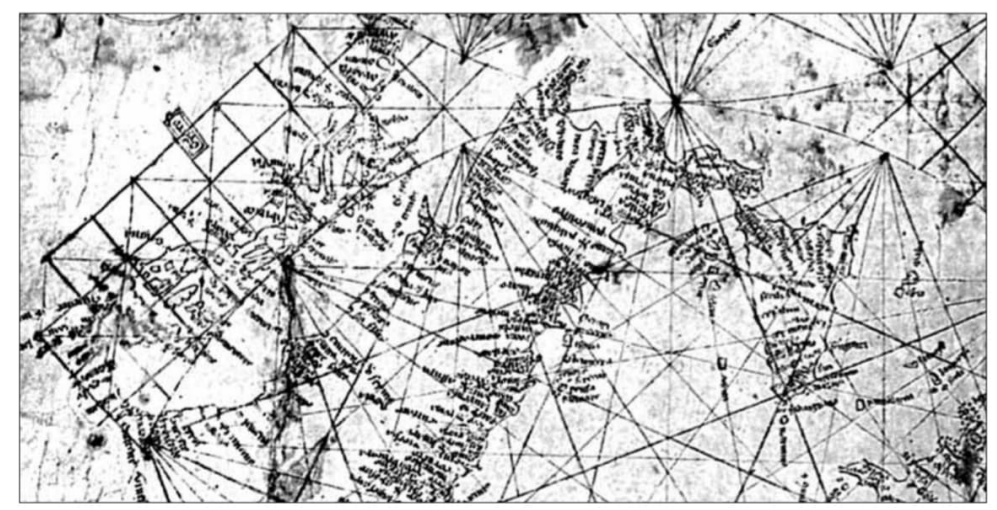

Figura 3. Anonimo. Portolano Pisano, $1290 \mathrm{ca}$. Bibliothèque Nationale de France, Cartes et Plans, Rés.Ge B 1118, particolare (immagine da Rivera Magos, Victor. «La chiave de tutta la Puglia», p. 68).

70 Santeramo, Salvatore. «Il Regio Secreto e il Mastro Portolano di Puglia in Barletta». Japigia, 1941, vol. XII, fasc. 4, pp. 225-239; BATTI, R. (ed.). Repertorio delle pergamene della Università o Comune di Barletta (1234-1658). Napoli: M. d'Auria, 1904, n. VII, pp. 7-17; Filangieri Di Candida, Riccardo. Codice Diplomatico Barese, X, Pergamene di Barletta del Regio Archivio di Napoli (1075-1309). Bari: Vecchi, 1927, n. 148, p. 258.

${ }_{71}$ Il testo originale dell'editto si crede sia andato perso a seguito del sacco turco del 1620. Questo però sopravvive grazie ad una trascrizione ora all'Archivio storico del comune di Manfredonia, mentre il relativo antigrafo si trovava iscritto nel Registro, Lib. F., ff. 230-231 dell'Archivio della Regia Zecca, anch'esso andato perso.

72 Rivera Magos, Victor. «I 'capitula' di Barletta e di Manfredonia (1297 e 1301). Due fonti fiscali per lo studio della Capitanata e della valle dell'Ofanto nel Medioevo». Bullettino dell'Istituto Storico Italiano per il Medioevo, 2018, vol. CXX, pp. 91-133. 
IL PORTO E LA STRADA. SPUNTI DI RIFLESSIONE SULLA TRADIZIONE

CONCERNENTE LA FONDAZIONE DI MANFREDONIA

ANTONIO PIO DI COSMO

\section{IL SITO DI FONDAZIONE ED I PROBLEMI CONCERNENTI LA COSTRUZIONE DELL'IDENTITÀ CITTADINA}

Occorre approfondire le implicazioni derivanti dalla previa costituzione di aggregati demici nell'area in cui sorgerà Manfredonia. Sappiamo che sin dalla prima decade del sec. XIII delle abitazioni si concentrano progressivamente attorno al nuovo porto, laddove la gente trova lavoro. In quegli stessi anni sorgono alloggi lungo la strata magna, ove già esiste un importante monastero e tutta una serie di piccole chiese come quella di S. Tommaso Apostolo, dipendenza dell'Abbazia della Trinità di Venosa, iuxta viam publicam ${ }^{73}$. Salendo verso Monte S. Angelo è poi possibile trovare una serie di orti e vigne appartenenti ai Templari ed agli Ospitalieri. In quest'ampia area sembrano collocarsi molte case, come quelle dimore oggetto delle attenzioni del re Carlo. Alcune di queste possono addirittura risalire ad una data successiva al 1156, in accordo con quanto testimoniato dal De Rebus Siculis, che attesta come i sipontini siano costretti a vivere sub vite et ficu ${ }^{74}$.

La progressiva espansione dell'aggregato demico attorno questi due punti focali sembra giustificare la formula del Datum Orte: in quo Civitas ipsa antiquitus fundata permanserat. Il lemma Civitas sembrerebbe alludere all'esistenza di una comunità, che nel lungo periodo ha sviluppato una propria identità collettiva ben definita, tanto da sollevare il redattore dal menzionare il nome della stessa. Ma cosa si intende? È possibile constatare l'esistenza di una vera e propria identità in quelle due aree demiche? Affermazione che suscita non poche perplessità.

Partendo da tali presupposti pare difficile sostenere la costruzione di un'identità cittadina, almeno a quella data. La sola presenza di edifici aggregati sui versanti della strata magna o situati sulla viciniore Ruga de Comite non si dimostra sufficiente a sviluppare un'identità comune degli abitanti. Un dato di fatto, che lascia propendere piuttosto per la semplice presa d'atto da parte del redattore del documento dell'esistenza nella sede eletta di una serie di abitazioni, che persistono lì da tempo.

Diversamene deve trattarsi la questione che concerne lo sviluppo di un'identità collettiva condivisa da coloro che vivono e lavorano attorno al porto di Cala dello Spontone. $\mathrm{Ma}$ fino a che punto questo nuovo porto, seppur centro nevralgico di attività, può essere considerato un fulcro di costruzione di identità? A maggior ragione se si tiene conto che ivi lavorano anche i genovesi ed i veneziani. Soggetti, che hanno un'identità ben definita ed un proprio console a cui fare riferimento. Questi vanno perciò esclusi a priori e non concorrono affatto a questo presunto processo di costruzione identitaria. A questi si aggiungono altre presenze alloctone come quelle di greci e slavi, che sono occupati nelle attività portuali. Se ne deduce che non può nemmeno parlarsi dell'esistenza di un'identità comune degli abitanti dell'area attorno al porto.

Nemmeno si comprende quale identità possa essere condivisa dai residenti di due aggregati di abitazioni distanti almeno 700 metri l'uno dall'altro. La distanza non sembra favorire quei legami di empatia e solidarietà tipici di una comunità, salvo i più profondi rapporti intercorrenti fra i soggetti coinvolti nelle attività del porto. Ciò è vero a maggior

73 Vitolo, Giovanni. Insediamenti cavensi in Puglia. Galatina: Congedo Editore, 1984, p. 77.

74 De Troia, Dalla distruzione, p. 32. 
ragione, se si considera che questa comunità frazionata sul territorio sembra pure priva di una serie di simboli suoi propri, che ne favoriscono il riconoscimento come tale. Sembra mancare pure una memoria comune. Indi per cui, i due raggruppamenti di abitazioni che si collocano a meno di un chilometro di distanza appaiono carenti di qualsivoglia elemento capace di agevolare un'omogeneizzazione dei soggetti che li abitano.

Bisogna perciò "correggere il tiro» e ricercare una serie di ulteriori fattori che possano agevolare la creazione di legami comunitari solidi. Si può intravedere un catalizzatore dei processi di omogeneizzazione nella liturgia greca, che viene celebrata a Manfredonia fino a quando l'arcivescovo Ginnasio, in cattedra dal 1586 al 1607, opera la riduzione dell'altare della Metropoli al rito latino. Un rito che in qualche modo accomuna gli autoctoni, i greci e gli slavi. Eppure il comune costume liturgico da solo non sembra bastare.

Il quadro sembra complicarsi, se si considera che in zona risiede una nutrita comunità ebraica. Costoro si occupano della concia delle pelli, di manifatture tessili, della salagione e conservazione di seppie e triglie, commercio dell'oro e attività di prestito. Una comunità che si insedia principalmente in quello che sarà il settore nord-orientale della nuova città, la già citata Ruga de Comite, area ricordata dalle fonti quale ex parte Iudeorum $^{75}$. Parecchie di queste famiglie sembrano progressivamente convertirsi al cristianesimo, almeno formalmente, per ottenere dagli Angioini, come già dagli Svevi, delle esenzioni fiscali. Nel 1294 i convertiti ammontano a 75 nuclei familiari. Ciò agevola l'omogeneizzazione culturale e favorisce la costruzione di un'identità civica. Cosa che avviene però tardi.

Non si deve poi dimenticare che la costruzione dell'identità civica si organizza a medio dell'aggregarsi di significati attorno a dei simboli della sfera civile e laica, nonché a quelli religiosi ed ecclesiastici. Si propone agli abitanti un insieme di segni, che assume maggior senso rispetto al contesto culturale in cui vengono adoperati. In particolare, deve considerarsi l'utilità del ricorso alla religione nei processi di creazione di una morfologia di autocoscienza civica, che si ancora al registro dei segni visivi connessi al culto del patrono locale, per agevolare la consapevolezza di una comune identità. Segni che divengono indispensabili per una risemantizzazione del passato e per una più efficace interpretazione del presente. In questo marco d'azione si comprende meglio il valore identitario afferito al culto locale per eccellenza: quello del santo patrono, che dai secc. vi-VII si coagula attorno ad un santo vescovo, predecessore del vescovo attuale, il quale proprio in quel periodo vede accrescere fortemente le proprie competenze ${ }^{76}$. Si può per-

75 Ognissanti, Pasquale. «Gli Ebrei a Manfredonia». La Capitanata, 1980-1982, voll. XXVII-XXVIII-XXIX, pp. 81-94; Ognissanti, Pasquale. "Sui primi insediamenti ebraici a Siponto». La Capitanata, 1985-1986, voll. XXI-XXII-XIII, pp. 209-218; OGnIssanti, Pasquale. Sui primi insediamenti ebraici a Siponto. La presenza ebraica nella civitas cristiana sipontina. Ipotesi di ricerca, Siponto e Manfredonia nella Daunia. Manfredonia, 1990; Ferorelli, Nicola. Gli Ebrei nell'Italia meridionale dall'età romana al secolo XVIII. Bologna: Forni, 1966, pp. 19-20; 221; Coniglio, Giuseppe. «Ebrei e cristiani novelli a Manfredonia nel 1534». Archivio Storico Pugliese, 1968, vol. XXI, fasc. 1-4; pp. 63-69; Summo, Giuseppina. Gli Ebrei in Puglia dall'XI al XVI secolo. Bari: Cressati, 1939, p. 48.

${ }_{76}$ Sul culto cittadino dei santi cfr: Orselli, Alba M. L’immaginario religioso della città medievale. Ravenna: Edizioni del Girasole, 1985; Orselli, Alba M. "Il santo patrono cittadino fra Tardo Antico e Alto 
tanto immaginare che la venerazione del patrono di Siponto, Lorenzo Maiorano (488?545? d.C.), sia stata usata come coagulante identitario. Tale ipotesi assume pregevolezza nell'assenza di più precise notizie circa segni laici o religiosi relativi ad un'epoca precoce.

Eppure, una tale devozione si radica solo con la progettazione della nuova cattedrale per ordine di Carlo I. Pertanto, si può parlare del culto del patrono come un catalizzatore di identità civica solo dal 1327, allorché si trasla in Manfredonia il corpo del vescovo Lorenzo, che viene collocato nella chiesa a lui dedicata. Una traslazione che ha un preciso scopo: estrapolare la leggenda laurenziana dal campo liturgico e dal tempo scandito dalla chiesa locale, per porla a fondamento della vita civile ed entro un più ampio progetto identitario e culturale, prospettato agli abitanti della nuova città. Il culto del santo vescovo stimola un processo di autoconsapevolezza storica degli abitanti. Se ne deduce che solo da un'epoca successiva alla traslazione è possibile parlare di una vera e propria identità civica, coagulata attorno alla tomba del santo patrono ed alla nuova cattedrale, centro dell'odierna vita cittadina. Nondimeno, si significa che il riferimento al patrono sipontino venuto da Oriente, da Costantinopoli per di più, permette ai greci di identificarsi ed, in un certo modo, coinvolge anche gli slavi, che hanno sviluppato un forte legame con la cultura romano orientale. Costoro sicuramente possono rivedersi in questo personaggio alloctono.

Deve poi precisarsi che l'assenza di ogni riferimento nel testo del Datum Orte al nome della città lascia pensare che tanto gli abitanti, quanto le autorità, come Manfredi, hanno consapevolezza di una precisa situazione di fatto. Quell'area abitata, che si va accrescendo attorno ai due catalizzatori demici del porto e della strata magna, non è nulla più di un'appendice di Siponto e non può essere percepita altrimenti in un'epoca così precoce.

I dati raccolti permettono di sostenere che un'identità civica vera e propria si solidifica solo fra la fine del sec. XIII e l'inizio del sec. XIV attorno al culto del patrono ed allorché la trama urbana assume compattezza. Un'identità che si rafforza nella prolungata convivenza, capace di omogenizzare le diverse etnie. Un processo che sembra agevolato in un certo qual modo anche dalla conversione vera o presunta degli ebrei.

Al contempo, pare ragionevole ritenere che il Datum Orte costituisca un esperimento, che tenta di recidere lo stretto legame con la città di Siponto, fungendo da catalizzatore per la costruzione di un'autonoma identità civica. Un esperimento però fallimentare. Come sottolineato da Martin, la realtà dinamica di Siponto le ha permesso di sopravvivere nel più lungo periodo, allorché «il sito è diventato inservibile», la città si è «spostata» e non è "scomparsa» ${ }^{77}$. Un mero spostamento comprovato dal fatto che il patrono rimane il vescovo Lorenzo, che funge da principale fattore nella strutturazione dell'identità civica.

Medioevo". In La cultura in Italia fra Tardo Antico e Alto Medioevo. Atti del Convegno, Roma 12-16 novembre 1979. Roma: Herder Editrice, 1981, pp. 413-435.

77 Martin, «La città di Siponto", pp. 31-32. 


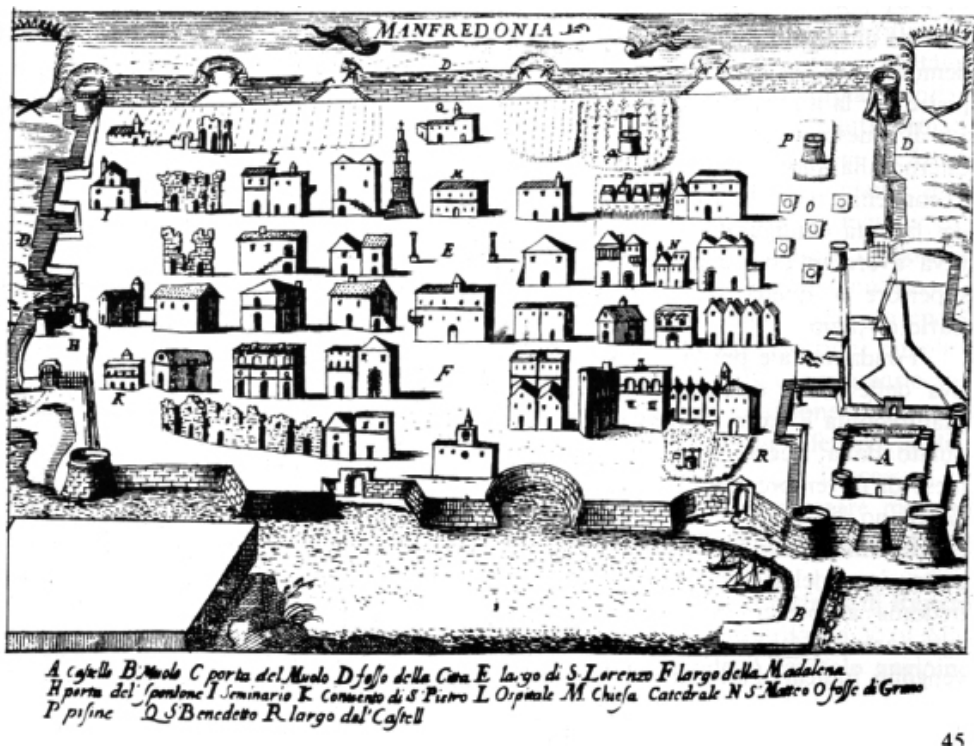

Figura 4. Mappa di Manfredonia, sec. xvi.

Consta notare l'assenza nelle fonti storico-letterarie prodotte nell'Italia meridionale sia coeve, sia immediatamente susseguenti al 1263 del toponimo «Manfredonia»; cosa che si riscontra pure nei documenti redatti sotto Manfredi. Persino il Datum Orte omette di menzionare il nome dell'aggregato demico. Ciò lascia dedurre che in quel momento il nucleo urbano non viene percepito come entità autonoma.

Il Datum Orte afferisce poi un ruolo fondamentale al conte Manfredi Maletta, titolare dell'Honor Montis Sancti Angeli e soprattutto proprietario di terre sulla strata magna che conduce a Monte Sant'Angelo ${ }^{78}$; terreni donatigli da papa Innocenzo IV prima del 1254. Sicché non si può escludere un intervento diretto del conte nella scelta del sito di fondazione. Costui, probabilmente, ha adibito le terre possedute presso l'asse viario ad uso abitativo.

Nel dicembre dello stesso anno la cancelleria pontificia emana per ordine di Bonifacio VIII un documento in favore di Maletta, laddove si menziona un solum, in quo terra que Manfredonia dicitur nunc extitit, su cui viene edificata la città che quam in perpetuam tui nominis memoriam Manfredoniam appellasti ${ }^{79}$. Il pontefice auspica la restituzione della

78 Palumbo, Pier Fausto. «Manfredi Maletta Camerario del Regno di Sicilia». In Palumbo, Pier Fausto (ed.). Contributi alla storia dell'età di Manfredi. Roma: Edizioni del Lavoro, 1959, pp. 166-172; 223.

79 Vendola, Documenti tratti dai registri vaticani, doc. 28, p. 28. 
città che è stata confiscata a Maletta nel 1271 da Carlo I e afferita con l'Honor e la contea di Lesina a Carlo principe di Salerno.

L'emanazione di un documento pontificio deve corroborare la posizione del conte, poiché le pressioni esercitate da Clemente IV non sono state sufficienti. Un tentativo andato a buon fine quello di Bonifacio VIII, che viene seguito da due provvedimenti emanati dalla cancelleria regia angioina nel 1300 e nel 1301. I documenti assicurano a Maletta, oltre alla castellania di Manfredonia, dei diritti sulle tratte del porto.

Questi documenti, in particolare quello pontificio, offrono una potente suggestione. I dati evinti hanno spinto Domenico Vendola a ipotizzare che il nome Manfredonia non onori il re Manfredi, ma piuttosto vada ricondotto all'azione di Manfredi Maletta. Ipotesi che si dimostra non del tutto pellegrina. Si può così sostenere la ragionevolezza di una fondazione nobiliare, nonostante questa sia una prerogativa regia. Nondimeno è possibile dimostrare numerose fondazioni signorili ed ecclesiastiche coeve, anche in un'epoca, come quella sveva ed angioina, connotata da un forte controllo monarchico. In tale ottica appare plausibile una fondazione operata dal Gran Camerario del Regno, su cui interviene il sovrano e nipote, che prevede privilegi ed esenzioni fiscali per essa. Eppure, tale assunto non convince del tutto. È più plausibile pensare ad un ruolo «ridotto» di Maletta, che funge da mero "catalizzatore» nello sviluppo del tessuto urbano da cui nasce Manfredonia. Si può ipotizzare che costui stimola degli insediamenti sulle sue terre, perché ubicate in un'ottima posizione come quella presso la strata magna. Un'ubicazione capace di fungere da attrattore per altre unità demiche. Nemmeno l'intervento di Maletta può essere qualificato come atto fondativo vero e proprio, tanto quanto non lo può essere quello di Manfredi, il quale deve limitarsi a corroborare un processo già in atto. Anzi Manfredi sembra intervenire solo a regolare una fase di insediamento del territorio già in stato avanzato, iniziata per così dire in modo spontaneo e irregolare nell'area limitrofa al porto. Questa va a sommarsi al progressivo adibirsi dei suoli divenuti proprietà di Maletta ad uso abitativo.

A seguito della guerra del Vespro si raffronta un'opera di revisione della memoria, che si realizza in modo "incrociato». Si vede concorrere sia la cancelleria pontificia, che vuole cancellare ogni traccia dell'intervento di Manfredi nella nascita della città, sia la stessa monarchia francese, la quale non ha interesse a far sopravvivere alcun consenso attorno al predecessore. A mezzo degli atti redatti dalle cancellerie si incide la memoria locale, creandone una alternativa, capace di distorcere quanto persiste del ricordo di Manfredi, senza però riuscire ad annullarlo. In assenza di una più cospicua documentazione non si può far altro che intuire il «trucco» messo in opera dai burocrati, quale sorta di falsificazione della memoria volta ad espungere quel personaggio scomodo.

L'operazione di revisione della memoria locale si dimostra precoce, offrendo prova di quanto il problema fosse sentito dalle autorità francesi. L'azione angioina anticipa il tentativo di ridefinizione posto in essere dalla cancelleria pontificia nel 1299. A testimoniarlo è frà Salimbene, il quale afferma che dopo il 1283 la città chiamata Manfredonia viene ridenominata: Sipontus nova ${ }^{80}$. La politica di Carlo d'Angiò condanna alla

80 Salimbene de Adam, Cronica, p. 685. 
damnatio quel presunto gesto di auto-magnificazione e si oppone all'uso dell'intitolazione a Manfredi. O, almeno, ciò ci fanno credere le cronache di Villani e Malispini.

\section{Conclusioni}

I dati concernenti la «nascita» di Manfredonia permettono di sostenere che quell'atto «unico»e «solenne» cantato dalla tradizione locale non si è mai verificato. Al contrario, sembra che la nuova città si costituisca come un progressivo addensarsi di presenze demiche attorno ai due punti nevralgici del porto di Cala dello Spuntone e della strata magna. Tale ricostruzione toglie pregevolezza al ruolo di Manfredi. Sembra più plausibile un intervento regio meno invasivo, che si riduce ad un tentativo di sistematizzazione di una realtà demica già esistente e sviluppatasi in modo spontaneo e disordinato. Quei nuclei demici vengono omogenizzati in una più ampia trama urbana e ricondotti entro gli schemi approntati per le cosiddette terrae novae.

I potenziali guadagni per il Regio Fisco, provenienti dal nuovo porto, spingono gli Angioini a completare non senza un qualche ripensamento l'ordito cittadino. Sopravvive però il problema politico connesso alla memoria di Manfredi. In tal senso si giustifica l'espressione di frà Salimbene, che ricorda come re Carlo ridenomina la città tentando di obliterare ogni legame con Manfredi.

\section{RIFERIMENTI BIBLIOGRAFICI}

Abulafia, David. Le due Italie. Relazioni commerciali tra il Regno normanno di Sicilia e i comuni dell'Italia settentrionale. Napoli: Guida Editori, 1991.

BARILE, Nicola Lorenzo. «Merci e mercati della Capitanata medievale: la testimonianza delle "pratiche di mercatura”" In Gravina, Armando (ed.). Atti del $33^{\circ}$ Convegno Nazionale sulla Preistoria, Protostoria, Storia della Daunia. San Severo: Centro Grafico S.r.l., 2013, pp. 175-188.

Batti, R. (ed.). Repertorio delle pergamene della Università o Comune di Barletta (1234-1658). Napoli: M. d'Auria, 1904.

Benvenuti de Rambaldis de Imola Comentum super Dantis Aldigherij Comoediam, Lacaita, James Philip (ed.). Firenze, 1887.

Bianchini, Ludovico. Storia delle finanze del Regno delle due Sicilie, De Rosa, Luigi (ed.). Napoli: Edizioni Scientifiche Italiane, 1971.

Breve chronicon De Rebus Siculis, Delle Donne, Fulvio (ed.). Roma: Istituto Storico Italiano per il Medioevo, 2017.

Carabellese, Francesco. Le relazioni commerciali fra la Puglia e la Repubblica di Venezia dal secolo $\mathrm{x}$ al xv. Bologna: Forni, 1991.

Carlucci, Rosanna. «I Diurnali di Matteo da Giovinazzo: una Cronaca del xiII secolo?». Studi Bitontini, 2003, vol. LXXV, pp. 41-58.

Cassandro, Giovanni. "I porti pugliesi nel medioevo». In Cassandro, Giovanni (ed.). Saggi di storia del diritto commerciale. Napoli: Edizioni Scientifiche Italiane, 1982, pp. 355-394.

Coniglio, Giuseppe. «Ebrei e cristiani novelli a Manfredonia nel 1534». Archivio Storico Pugliese, 1968, vol. XXI, fasc. 1-4; pp. 63-69. 
Cristiani, Emilio. "I più recenti orientamenti sulla "Storia fiorentina" malispiniana». Bolletino Storico Pisano, 2001, vol. LXX, pp. 305-310.

De Troia, Giuseppe. Dalla distruzione di Siponto alla fortificazione di Manfredonia. Fasano: Schena, 1985.

De Troia, Giuseppe. Foggia e la Capitanata nel Quaternus excadenciarum di Federico II di Svevia. Fasano: Schena, 1994.

De Troia, Giuseppe. «Gli annotamenti di Matteo Spinelli da Giovinazzo: frammenti di cronaca del XIII secolo, osservazioni critiche». La Capitanata, 2001, vol. XXVIII, pp. 167-205.

Del Trepro, Mario. «Stranieri nel Regno di Napoli. Le élites finanziarie e la strutturazione dello spazio economico e politico». In Rossetti, Gabriella (ed.). Dentro la città. Stranieri e realtà urbane nell'Europa dei secoli XI-XV. Napoli: Liguori, 1989, pp. 179-233.

Del Trepro, Mario. "Federico II e il Mediterraneo». Studi Storici, 1996, vol. XXXVII, pp. 373390.

Del Treppo, Mario. «Prospettive mediterranee della politica economica di Federico II». In Esch, Arnold e Kamp, Norbert (edd.). Friedrich II. Tagung des Deutschen Historischen Instituts in Rom im Gedenkjahr. Tübingen: Bibliothek des Deutschen Historischen Instituts in Rom, 1996, pp. 316-338.

Delano Smith, Chatrine. «Tipi di insediamenti nella zona costiera di Foggia». In Civiltà preistoriche e protostoriche della Daunia. Firenze: Istituto Italiano di Preistoria e Protostoria, 1975, pp. 5-15.

Delano Smith, Chatrine. Daunia vetus. Napoli: Tipografia Laurenziana, 1978.

Delle Donne, Fulvio. «Tradizioni testuali e (ri)scrittura della storia. Il caso del Breve chronicon de rebus Siculis». Zeitschrift Quellen und Forschungen aus Italienischen Archiven und Bibliotheken Band, 2016, vol. XCVI, pp. 174-190.

Ferorelli, Nicola. Gli Ebrei nell'Italia meridionale dall'età romana al secolo XVIII. Bologna: Forni, 1996.

Filangieri Di Candida, Angerio. «Le fondazioni di città nello sviluppo degli insediamenti del Mezzogiorno». Working Paper, 2007, vol. XII, fasc. 1, pp. 23-24.

Filangieri Di Candida, Riccardo. Codice Diplomatico Barese, X, Pergamene di Barletta del Regio Archivio di Napoli (1075-1309). Bari: Vecchi, 1927.

Francesco Balducci Pegolotti. La pratica della mercatura, Evans, Allan (ed.). Cambridge Mass: Medieval Academy of America, 2012.

GAY, Giulio. L'Italia meridionale nell'impero bizantino. Bologna: Forni, 1978.

Giagnacovo, Maria. Appunti di metrologia mercantile genovese: Un contributo della documentazione aziendale Datini. Firenze: Firenze University Press, 2014.

Giovanni Villani. Nuova cronica, Porta, Giuseppe (ed.). Parma: Ugo Guanda Editore, 19901991.

Giunta, Francesco. «Manfredi e Manfredonia». In Giunta, Francesco (ed.). La coesistenza nel Medioevo. Bari: Dedalo, 1968.

Historia diplomatica Friderici Secundi, Huillard-Bréholles, Jean-Louis-Alphonse (ed.). Paris: Plon, 1852-1861.

Il registro della cancelleria di Federico II del 1239-1240, Carbonetti Venditelli, Cristina (ed.). Roma: Istituto Storico Italiano per il Medio Evo, 2002.

Ioannis Cinnami Epitome rerum ab Ioanne et Alexio Comnenis gestarum, Meineke, A. (ed.). Corpus Scriptorum Historiae Byzantinae. Bonn: Weber, 1836.

Iorio, Raffaele. «Siponto, Canne». In MusCA, Giosuè (ed.). Itinerari e centri urbani nel Mezzogiorno normanno-svevo. Atti delle decime giornate normanno-sveve. Bari, 21-24 ottobre 1991. Bari: Dedalo, 1993, pp. 385-425. 
Johannes Victoriensis. Chronicon Carinthiae, Böhmer, J. F. (ed.). Fontes Rerum Germanicarum. Stuttgart: Verlag, 1843-1868.

Laganara, Caterina. "In civitate sipontina... lo spazio urbano e le dinamiche insediative». In LAganara, Siponto. Archeologia di una città abbandonata nel Medioevo. Foggia: Grenzi Editore, 2011, pp. 47-53.

Licinio, Raffaele. Masserie medievali. Masserie, massari e carestie da Federico II alla Dogana delle pecore. Bari: Adoa, 1989.

Licinio, Raffaele. «La normativa sul sistema masseriale». In MusCA, Giosuè (ed.). Le eredità normanno-sveve nell'età angioina. Persistenze e mutamenti nel Mezzogiorno. Atti delle quindicesime giornate normanno-sveve. Bari, 22-25 ottobre 2002. Bari: Dedalo, 2004, pp. 197-218.

Licinio, Raffaele. "I poteri territoriali: re, signori, vescovi e città». In SALvemini, Biagio e MassAFRA, Angelo (edd.). Storia della Puglia. Dalle origini al Seicento. Roma-Bari: Laterza, 2005, pp. 130-149.

Martin, Jean Marie. La Pouille du vI a au XII siècle. Roma: École Française de Rome, 1993.

Martin, Jean Marie. Les actes de l'abbaye de Cava concernant le Gargano. Bari: Società di Storia Patria per la Puglia, 1994.

Martin, Jean Marie. «La città di Siponto nei secoli XI-XIII». In Houben, Hans (ed.). San Leonardo di Siponto. Cella monastica, canonica, "domus Theutonicorum". Atti del Convegno internazionale, Manfredonia, 18-19 marzo 2005. Galatina: Congedo Editore, 2006, pp. 15-32.

Martin, Jean Marie. La vita quotidiana nell'Italia Meridionale ai tempi dei Normanni. Torino: Bur, 2018.

Martin, Jean Marie e NoyÉ, Ghislaine. «Les campagnes de l'Italie méridionale byzantine ( $\mathrm{x}^{\mathrm{e}}-\mathrm{XI}^{\mathrm{e}}$ siècles)». Mélanges de l'École Française de Rome - MoyenAge, 1989, vol. CI, pp. 558-596.

Mastrodd, Laura. "Contributo al testo critico della Storia fiorentina di Ricordano Malispini». Bullettino dell'Istituto Storico Italiano per il Medioevo, 2000-2001, vol. CIII, pp. 239-293.

Morelli, Serena. "Una fonte inedita per la storia del Regno di Napoli in età angioina: le carte di Leon Cadier». Rassegna degli Archivi di Stato, 1997, voll. II-III, pp. 471-474.

Nebort, René. «Les basses terrasses alluviales, témoins de l'occupation des sols. Italie méridionale, Sicile». In Noyé, Ghislaine (ed.). Structures de l'habitat et occupation du sol dans les pays méditerranéens: les méthodes et l'apport de l'archéologie extensive. Actes de la rencontre organisée par l'École Française de Rome, Paris, 12-15 novembre 1984. Rome-Madrid: École Française de Rome-Casa de Valázquez, 1988, pp. 401-405.

Ognissanti, Pasquale. "Gli Ebrei a Manfredonia». La Capitanata, 1980-1982, voll. XXVII-XXVIII-XXIX, pp. 81-94.

Ognissanti, Pasquale. «Il porto di Siponto e di Manfredonia». La Capitanata, 1984-1985, vol. XLII, pp. 9-51.

Ognissanti, Pasquale. «Sui primi insediamenti ebraici a Siponto». La Capitanata, 1985-1986, voll. XXI-XXII-XIII, pp. 209-218.

Ognissanti, Pasquale. "La storiografia sipontina». La Capitanata, 1987, vol. XXVI, pp. 1-36.

Ognissanti, Pasquale. Sui primi insediamenti ebraici a Siponto. La presenza ebraica nella civitas cristiana sipontina. Ipotesi di ricerca, Siponto e Manfredonia nella Daunia. Manfredonia, 1990.

Orselli, Alba M. «Il santo patrono cittadino fra Tardo Antico e Alto Medioevo». In La cultura in Italia fra Tardo Antico e Alto Medioevo. Atti del Convegno, Roma 12-16 novembre 1979. Roma: Herder Editrice, 1981, pp. 413-435.

Orselli, Alba M. L'immaginario religioso della città medievale. Ravenna: Edizioni del Girasole, 1985.

Palmieri, Stefano. «Bartolommeo Capasso e l'edizione delle fonti storiche napoletane». Napoli Nobilissima, 2001, vol. III, pp. 147-162. 
CONCERNENTE LA FONDAZIONE DI MANFREDONIA

ANTONIO PIO DI COSMO

Palumbo, Pier Fausto. «Honor Montis Sancti Angeli». Archivio Storico Pugliese, 1953, vol. IV, pp. 304-370.

Palumbo, Pier Fausto. «La fondazione di Manfredonia». Archivio Storico Pugliese, 1953, vol. IV, pp. 371-407.

Palumbo, Pier Fausto. "Manfredi Maletta Camerario del Regno di Sicilia». In Palumbo, Pier Fausto (ed.). Contributi alla storia dell'età di Manfredi. Roma: Edizioni del Lavoro, 1959, pp. 109-225.

Palumbo, Pier Fausto. Contributi alla storia dell'età di Manfredi. Roma: Edizioni del Lavoro, 1959.

Poleggi, Ennio. «La costruzione della città portuale, un nuovo tema di storia». In Poleggi, Ennio (ed.). Città portuali del Mediterraneo. Storia e archeologia. Atti del Convegno Internazionale Città portuali del Mediterraneo, Genova, 1985. Genova: Sagep Editrice, 1989, pp. 7-10.

PORTA, Giuseppe. «Le varianti redazionali come strumento di verifica dell'autenticità dei testi: Villani e Malispini». In Guida, Saverio e Latella, Fortunata (edd.). La filologia romanza e $i$ codici. Atti del Convegno della società italiana di filologia romanza, Messina 12-19 dicembre 1991. Messina: Signa, 1993, pp. 481-487.

Prencipe, Domenico. «Leconomia di Manfredonia nel periodo protoangioino». Bollettino del Nuovo Centro di Documentazione Storica, 2001-2004, vol. VI, pp. 201-220.

Pryor, John H. «Foreign policy and economic policy: the Angevins of Sicily and the economic decline of Southern Italy». In Frappell, L.O. (ed.). Principalities, powers and estates studies in medieval and early modern government and society. Adelaide: Adelaide University Union Press, 1980, pp. 43-55.

Quaternus de excadenciis et revocatis Capitinatae de mandato imperialis maiestatis Friderici secundi, Amelli, A. (ed). Montecassino: San Paolo, 1903.

Regesto di San Leonardo di Siponto. Camobreco, Fortunato (ed.). Regesta Chartarum Italiae 10. Roma: Istituto Storico Italiano, 1913.

Ricordano Malispini. Istoria fiorentina, Muratori, Ludovico A. (ed.). Rerum Italicarum Scriptores, VII. Milano, 1726.

Rivera Magos, Victor. «La chiave de tutta la Puglia. Presenze straniere, attività commerciali e interessi mediterranei a Manfredonia, 'agriporto' di Capitanata (secoli XIII-XVI)». In Licinio, Raffaele (ed.). Storia di Manfredonia. Il Medioevo. Bari: Edipuglia, 2008, pp. 63-99.

Rivera Magos, Victor. «I 'capitula' di Barletta e di Manfredonia (1297 e 1301). Due fonti fiscali per lo studio della Capitanata e della valle dell'Ofanto nel Medioevo». Bullettino dell'Istituto Storico Italiano per il Medioevo, 2018, vol. CXX, pp. 91-133.

Salimbene de Adam. Cronica, Scalia, Giuseppe (ed.). Roma-Bari, Laterza, 1966.

SANFILIPpo, Mario. "Le città pugliesi dall'xi al Xv secolo: continuità e persistenze negli insediamenti difensivi». In SAnfilippo, Mario (ed.). Medioevo e città nel regno di Sicilia e nell'Italia comunale. Messina: Sicania, 1991, pp. 37-53.

Santeramo, Salvatore. «Il Regio Secreto e il Mastro Portolano di Puglia in Barletta». Japigia, 1941, vol. XII, fasc. 4, pp. 225-239.

Sarcinelli, Giuseppe. "La collezione numismatica del Comune di Manfredonia». In MazzeI, Marina (ed.). Siponto antica. Foggia: Grenzi Editore, 1999, pp. 77-84.

Sarcinelli, Giuseppe. Prima e dopo Manfredi. Monete tra Siponto e Manfredonia nella collezione civica. Foggia: Edizioni del Rosone, 2010.

Sarcinelli, Giuseppe. «Le monete». In Laganara, Caterina (ed.). Siponto. Archeologia di una città abbandonata nel Medioevo. Foggia: Grenzi Editore, 2011, pp. 197-206.

Sarcinelli, Giuseppe. "Moneta reale, moneta di conto: tra fonti materiali e fonti scritte». In Laganara, Caterina (ed.). L'abitare: spazi domestici e quadri di vita. Dalla ricerca archeologica nella Siponto medievale. Foggia: Grenzi Editore, 2012, pp. 121-136. 
Sarnelli, Pompeo. Cronologia De’ Vescovi et Arcivescovi Sipontini. Manfredonia: Stamperia Arcivescovile, 1680.

Schmiedt, Giulio. «Contributo della fotografia aerea alla ricostruzione della antica laguna compresa tra Siponto e Salapia». Archivio Storico Pugliese, 1973, vol. XXVI, pp. 159-172.

Serricchio, Cristanziano. "Manfredi e la fondazione di Manfredonia». Archivio Storico Pugliese, 1972, vol. XXV, pp. 483-509.

Spineldi, Matteo. Diurnali, Vigo, G. (ed.). Napoli: G. Dura, 1872.

Stipo, Pasquale. I "Notamenti" di Messer Matteo Spinelli da Giovenazzo, tra vero e falso, frammenti di cronaca del $13^{\circ}$ secolo (I Diurnali). Bisceglie: Eurografica, 2013.

Summo, Giuseppina. Gli Ebrei in Puglia dall'XI al XVI secolo. Bari: Cressati, 1939.

TABACCo, Giovanni. "Il potere politico nel Mezzogiorno d'Italia dalla conquista normanna alla dominazione aragonese». In Il Mezzogiorno medievale nella storiografia del secondo dopoguerra: risultati e prospettive. Atti del IV Convegno dell'Associazione dei Medioevalisti Italiani, Università di Calabria, 1982. Cosenza: Rubbettino, 1985, pp. 65-111.

Ughelli, Ferdinando. Italia Sacra. Venezia: Forni, 1721.

Vendola, Domenico. Documenti tratti dai registri vaticani da Bonifacio VIII a Clemente V. Trani: Vecchi, 1963.

Violante, Francesco. «Da Siponto a Manfredonia: note sulla "fondazione”". In Licinio, Raffaele (ed.). Storia di Manfredonia. Il Medioevo. Bari: Edipuglia, 2008, pp. 9-24.

Violante, Francesco. "Organizzazione del territorio e strutture produttive tra XI e XVI secolo". In Licinio, Raffaele (ed.). Storia di Manfredonia. Il Medioevo. Bari: Edipuglia, 2008, pp. 101-123.

Vitolo, Giovanni. Insediamenti cavensi in Puglia. Galatina: Congedo Editore, 1984.

Zazzaretta, Alessandro. «Sui Diurnali di Matteo Spinelli. Premessa per un riesame della questione spinelliana». Archivio Storico Pugliese, 1970, vol. XXIII, pp. 199-214. 